\title{
A Signaled Locomotor Avoidance Action Is Fully Represented in the Neural Activity of the Midbrain Tegmentum
}

\author{
${ }^{(D)}$ Sebastian Hormigo, Bharanidharan Shanmugasundaram, Ji Zhou, and ${ }^{\circledR}$ Manuel A. Castro-Alamancos \\ Department of Neuroscience, University of Connecticut School of Medicine, Farmington, Connecticut 06001
}

Animals, including humans, readily learn to avoid harmful and threatening situations by moving in response to cues that predict the threat (e.g., fire alarm, traffic light). During a negatively reinforced sensory-guided locomotor action, known as signaled active avoidance, animals learn to avoid a harmful unconditioned stimulus (US) by moving away when signaled by a harmless conditioned stimulus (CS) that predicts the threat. CaMKII-expressing neurons in the pedunculopontine tegmentum area (PPT) of the midbrain locomotor region have been shown to play a critical role in the expression of this learned behavior, but the activity of these neurons during learned behavior is unknown. Using calcium imaging fiber photometry in freely behaving mice, we show that PPT neurons sharply activate during presentation of the auditory CS that predicts the threat before onset of avoidance movement. PPT neurons activate further during the succeeding CS-driven avoidance movement, or during the faster US-driven escape movement. PPT neuron activation was weak during slow spontaneous movements but correlated sharply with movement speed and, therefore, with the urgency of the behavior. Moreover, using optogenetics, we found that these neurons must discharge during the signaled avoidance interval for naive mice to effectively learn the active avoidance behavior. As an essential hub for signaled active avoidance, neurons in the midbrain tegmentum process the conditioned cue that predicts the threat and discharge sharply relative to the speed or apparent urgency of the avoidance (learned) and escape (innate) responses.

Key words: escape; avoidance; goal-directed behavior; midbrain; basal ganglia; motor plan

\section{Significance Statement}

During signaled active avoidance behavior, subjects move away to avoid a threat when directed by an innocuous sensory stimulus. Using imaging methods in freely behaving mice, we found that the activity of neurons in a part of the midbrain, known as the pedunculopontime tegmentum, increases during the presentation of the innocuous sensory stimulus that predicts the threat and also during the expression of the learned behavior as mice move away to avoid the threat. In addition, inhibiting these neurons abolishes the ability of mice to learn the behavior. Thus, neurons in this part of the midbrain code and are essential for signaled active avoidance behavior.

\section{Introduction}

Animals, including humans, innately respond to unconditioned stimuli (US) by seeking (approaching), freezing, or fleeing (escaping) depending on the circumstances. Given the opportunity, escaping usually occurs in the presence of harm (painful foot-shock or loud stimulus) or an approaching predator

\footnotetext{
Received Jan. 6, 2021; revised Mar. 2, 2021; accepted Mar. 16, 2021.

Author contributions: S.H., B.S., J.Z., and M.A.C.-A. performed research; S.H., B.S., J.Z., and M.A.C.-A. analyzed data; S.H., B.S., J.Z., and M.A.C.-A. edited the paper; M.A.C.-A. designed research; M.A.C.-A. wrote the first draft of the paper; M.A.C.-A. wrote the paper.

This work was supported by National Institutes of Health grants to M.A.C.-A. We thank Dorian Chabbert for technical assistance.

The authors declare no competing financial interests.

Correspondence should be addressed to Manuel A. Castro-Alamancos at mcastro@uchc.edu.

https://doi.org/10.1523/JNEUROSCI.0027-21.2021

Copyright $\odot 2021$ the authors
}

(looming stimulus), and the neural circuits responsible for these innate defensive reactions have received significant attention in several species (Brandao et al., 1994; Blanchard et al., 2005; Yilmaz and Meister, 2013; Xiong et al., 2015; Peek and Card, 2016; Tovote et al., 2016; Terburg et al., 2018; Branco and Redgrave, 2020; Hersman et al., 2020). In addition, animals readily learn to cope with harmful and threatening situations that are predictable and/or controllable. For instance, crossing a busy street is dangerous, but humans cope with this situation very effectively by relying on learned signals (streetlights) that predict the danger. Similarly, in the laboratory, during a negatively reinforced sensory-guided locomotor behavior, known as signaled active avoidance, rodents learn to avoid a harmful US by moving away (e.g., shuttling in a cage) during an interval signaled by a harmless conditioned stimulus (CS) (Mowrer, 1960; Bolles, 1970; Mineka, 1979; LeDoux et al., 2017). 
Although signaled active avoidance has been studied for almost a century, the basic neural circuits mediating the expression of this behavior have remained elusive. Forebrain circuits, including PFC, amygdala, and striatal nuclei, may be important for acquiring and modulating certain forms of avoidance behavior (Amorapanth et al., 2000; Bravo-Rivera et al., 2014; Ramirez et al., 2015), but midbrain circuits can mediate expression of signaled active avoidance (Cohen and Castro-Alamancos, 2007, 2010a,b). Recently, an area in the midbrain, the pedunculopontine tegmentum (PPT), which together with the cuneiform nucleus forms the midbrain locomotor region (Ryczko and Dubuc, 2013; Roseberry et al., 2016; Capelli et al., 2017; Mena-Segovia and Bolam, 2017; Arber and Costa, 2018), has been shown to be critically involved in the expression of signaled active avoidance (Hormigo et al., 2019). Optogenetic inhibition of CaMKII PPT neurons (termed hereafter PPT neurons, for simplicity) abolishes active avoidance responses driven by the CS, without impeding escape responses driven by the US (Hormigo et al., 2016, 2019, 2020a). Conversely, frequency-dependent optogenetic excitation of these PPT neurons drives locomotor responses at speeds spanning from slow avoidance responses to very fast escape responses (Hormigo et al., 2019). However, the activity of PPT neurons during signaled active avoidance behavior is not known.

Using calcium imaging fiber photometry in freely behaving mice, we show that the activity of PPT neurons fully represents signaled active avoidance by encoding the CS signal before the avoidance movement and both the conditioned (avoidance) and unconditioned (escape) movement responses driven by the CS and US, respectively.

\section{Materials and Methods}

Experimental design and statistical analysis. All procedures were reviewed and approved by the institutional animal care and use committee and conducted in adult $(>8$ weeks) male and female mice. The results from both sexes were combined since there is no sex difference in the behavior measured for the strains used (Hormigo et al., 2019).

All experiments involved a repeated-measures design in which the mice or cells serve as their own controls. Unless otherwise stated, conclusions are based on within-subject comparisons. We tested for a main effect using a two-way mixed design ANOVA followed by comparisons with Tukey's test. In the mixed design ANOVA, the repeated-measures factor was the main effect (with as many levels as conditions tested) and the other factor was the animals' sessions (behavior). The sessions factor is statistically independent because the fully trained animals must perform the signaled active avoidance trials (auditory CS trials) at the same high level per session (by definition, one session has no effect on task performance on the other sessions). Tukey tests were conducted for the repeated-measures factor when the within-subjects effect ( $F$ value) was statistically significant at a level of $p<0.01$. Unless otherwise noted, all comparisons are between conditions within the same session.

The experiment timeline included the following sequence of phases: adeno-associated viral (AAV) vector injections, optical fiber implantation, active avoidance learning, active avoidance testing, and histology. The active avoidance learning or testing phase commenced 5-6 weeks after AAV injections.

To enable rigorous approaches, we maintain a local server with a central database accessed through a wiki that logs all details and metadata related to the experiments, including all information about animals and details about surgical procedures, behavioral sessions, electrophysiological recordings, histology, and scripts used for analyses. Moreover, during daily behavioral sessions, computers run experiments automatically using preset parameters logged for reference during analysis. Analyses are performed using scripts that automate all aspects of data analysis from access to metadata and data files to population statistics and graph generation (scripts and metadata will be accessible through our website or by request).

Strains and AAVs. The following AAVs (injected undiluted) and mouse strains were used in the present study. To measure calcium signals in CaMKII PPT neurons, we injected C57BL/6J mice (Jax 00664) with AAV5-CaMKII-GCaMP6f-WPRE-SV40 (UPenn Vector core, titers: $\left.1.53 \times 10^{13} \mathrm{GC} / \mathrm{ml}\right)$. To inhibit CaMKII PPT neurons with optogenetics (green light), we injected C57BL/6J mice with AAV5-CaMKIIaeArchT3.0-EYFP (UNC vector core; titers: $4 \times 10^{12}$ virus molecules $/ \mathrm{ml}$ ).

Surgeries. Fiber photometry experiments involved unilaterally injecting $0.4 \mu \mathrm{l}$ of AAV during isoflurane anesthesia $(\sim 1 \%)$. Subsequently, a single optical fiber $(400 \mu \mathrm{m}$ in diameter) was implanted unilaterally and held in place with a combination of screws, cyanoacrylate, and dental cement. A fitted thin cap of black aluminum foil was placed inside the dental cement to block any room light from reaching the optical fiber. Optogenetics experiments involved bilaterally injecting $0.3 \mu \mathrm{l}$ AAV per site and subsequently implanting a dual optical fiber $(200 \mu \mathrm{m}$ in diameter). Animals received carprofen after surgery. The stereotaxic coordinates for AAV injections and optical fiber implantations (in $\mathrm{mm}$ from bregma; lateral from the midline; ventral from the bregma- $\lambda$ plane) in PPT: 4.7 posterior; 1.25 lateral; 3.1 ventral. The injection cannula and optical fibers were inserted at a $20^{\circ}$ angle in the posterior direction.

Fiber photometry. We used a 2-channel (465 and $405 \mathrm{~nm}$ ) fiber photometry system (Doric Lenses) with alternating (20-60 and 20-50 $\mu \mathrm{W}$, respectively) pulses of light excitation at $100 \mathrm{~Hz}$ (per each $10 \mathrm{~ms}, 465$ is on for $3 \mathrm{~ms}$, and $2 \mathrm{~ms}$ later 405 is on for $3 \mathrm{~ms}$ ). The emission peak signals (525 and $430 \mathrm{~nm}$ for GCaMP6f and control emissions) evoked by the $465 \mathrm{~nm}$ and 405 pulses were acquired at $20 \mathrm{kHz}$ and measured at the end of each pulse. To calculate $\mathrm{F}_{\mathrm{o}}$, the 430 signal was scaled to the 525 signal (F) using the slope of the linear fit. Finally, $\mathrm{F} / \mathrm{F}_{\mathrm{o}}$ was calculated with the following formula: $\left(\mathrm{F}-\mathrm{F}_{\mathrm{o}}\right) / \mathrm{F}_{\mathrm{o}}$. Because of the nature of the behavior studied, a swivel is essential. Our swivel contains a mechanism that locks it in place during recording periods used for analysis. Every few trials, the swivel was allowed to rotate (to relieve minor strain) during controlled periods of the intertrial intervals (ITIs), which were excluded from all analyses. Thus, all data excludes rotation of the swivel. In addition, an aluminum cap was implanted during surgery to assure that ambient light was not leaking into the implant and reaching the optical fiber; this was tested in each animal by comparing fluorescence signals in the dark versus normal cage illumination.

Optogenetics. The implanted dual optical fibers were connected to patch cables using sleeves. A black aluminum cap covered the head implant and completely blocked any light exiting at the ferrule's junction. Furthermore, the experiments occurred in a brightly lit cage that made it difficult to detect any light escaping the implant. The other end of the patch cables was connected to a dual light swivel (Doric Lenses) that was coupled to a green laser $(520 \mathrm{~nm} ; 100 \mathrm{~mW})$ to activate Arch. The electrophysiological effects of optogenetic green light (continuous) were previously characterized using both whole-cell recordings in slices and single-unit recordings in anesthetized mice in vivo (Hormigo et al., 2016, 2019). Unless otherwise noted, the behavioral experiments used green light between 25 and $35 \mathrm{~mW}$. Power is regularly measured by flashing the connecting patch cords onto a light sensor with the sleeve on the ferrule.

Active avoidance task in a shuttle box. Mice were trained in the active avoidance task using procedures similar to those described previously for rats and mice (Cohen and Castro-Alamancos, 2007, 2010b; Hormigo et al., 2016, 2019). During an active avoidance session, mice are placed in a standard shuttle box (16.1 inch $\times 6.5$ inch) that has two compartments separated by a partition with side walls forming a doorway that the animal has to traverse to shuttle between compartments. A trial consists of a $7 \mathrm{~s}$ avoidance interval followed by a $10 \mathrm{~s}$ escape interval. During the avoidance interval, an auditory CS is presented for the duration of the interval or until the animal produces a conditioned response (avoidance or avoid response) by moving to the adjacent compartment, whichever occurs first. If the animal avoids by moving to the next compartment, the CS ends, the escape interval is not presented, and the trial terminates. However, if the animal does not avoid, the escape interval ensues presenting white noise and a mild scrambled electric foot-shock 
$(0.3 \mathrm{~mA})$ delivered through the grid floor of the occupied half of the shuttle box. This US readily drives the animal to move to the adjacent compartment (escape response), at which point the US terminates, and the escape interval and the trial ends. Thus, an avoidance or avoid response will eliminate the imminent presentation of a harmful stimulus. An escape response is driven by presentation of the harmful stimulus to eliminate the harm it causes. In principle, successful avoidance is highly adaptive because it warrants the absence of harm. Each trial is followed by an ITI (duration is randomly distributed; $25-45 \mathrm{~s}$ range), during which the animal awaits the next trial.

Active avoidance task variations. We used two different basic signaled active avoidance tasks (AA1 and AA2) based on the consequence of shuttling during the ITI (intertrial crossings). In AA1, mice were free to cross between compartments, and there was no consequence for intertrial crossings. In AA2, mice receive a $0.2 \mathrm{~s}$ foot-shock $(0.3 \mathrm{~mA})$ and white noise for each intertrial crossing. Therefore, in AA2, mice must passively avoid during the ITI by inhibiting their tendency to shuttle during active exploration between trials. AA2 tests signaled active avoidance during the signaled avoidance interval and unsignaled passive avoidance during the unsignaled ITI. In AA1 and AA2, the CS is an $8 \mathrm{kHz}$ tone at $81 \mathrm{~dB}$.

In addition, we used a CS discrimination task (AA3) in which mice must respond differently to a CS1 $(8 \mathrm{kHz}$ tone at $81 \mathrm{~dB})$ and a CS2 $(4 \mathrm{kHz}$ tone at $68 \mathrm{~dB})$ presented randomly. Thus, animals perform regular signaled active avoidance to CS1 (e.g., AA1 or AA2) but perform signaled passive avoidance to CS2; intertrial crossings are not punished. In AA3, if animals shuttle during the CS2 avoidance interval, they receive a $0.5 \mathrm{~s}$ foot-shock $(0.3 \mathrm{~mA})$ with white noise and the trial ends. If animals do not shuttle during the CS2 avoidance interval, the CS2 trial terminates at the end of the avoidance interval (i.e., successful signaled passive avoidance)

Finally, we also used a signaled active avoidance task with different avoidance interval durations that are signaled by different CS (AA4). Three different CS, CS1 $(8 \mathrm{kHz}$ tone at $81 \mathrm{~dB}), \mathrm{CS} 2(10 \mathrm{kHz}$ tone at $82 \mathrm{~dB})$, and CS3 $(12 \mathrm{kHz}$ tone at $82 \mathrm{~dB})$, signal a different avoidance interval duration of 4,7 , and $15 \mathrm{~s}$, respectively. Otherwise, this task is identical to AA2 (i.e., mice are punished for producing intertrial crossings). In AA4, mice adjust their response latencies according to the duration of the avoidance interval signaled by each CS.

Behavioral measures and video tracking in the shuttle box. There are three main variables representing task performance in the shuttle box. The percentage of active avoidance responses (\% avoids) represents the trials in which the animal actively avoided the US in response to the CS. The response latency (latency) represents the time (seconds) at which the animal enters the safe compartment after the CS onset; avoidance latency is the response latency only for successful active avoidance trials (excluding escape trials). The number of crossings during the ITI (intertrial crossings) represents either random shuttling because of locomotor activity in the AA1 and AA3 procedures, or failures to passively avoid in the $\mathrm{AA} 2$ and $\mathrm{AA} 4$ procedure.

Animals are also video tracked (30 FPS) during active avoidance sessions. The tracking followed color markers located on the head connector above the nose and between the ears. Several movement (tracking) measures were derived during active avoidance. Distance was the number of pixels crossed by the animal in its trajectory during the avoidance and escape intervals of a trial (trial distance) or during the ITI (intertrial distance). Trial speed was the trial distance divided by the response latency. Intertrial speed was the intertrial distance divided by the ITI duration. Trial velocity was the displacement divided by the response latency (displacement was the number of pixels in a straight line between the position of the animal at trial start and the position of the animal at trial end when the animal avoided or escaped). Pixel measures were converted to metric units using calibrations. Trial speed and intertrial speed represent the overall movement of the animal in any direction during those periods, whereas trial velocity represents movement in the correct direction to avoid.

Histology. Mice were deeply anesthetized with an overdose of ketamine. Upon losing all responsiveness to a strong tail pinch, the animal was decapitated, and the brain was rapidly extracted and placed in fixative. The brain was sectioned (100 $\mu \mathrm{m}$ sections) in the coronal or sagittal planes. Sections were mounted on slides, coverslipped with DAPI mounting media, and photographed using a fluorescent microscope.

\section{Results}

\section{PPT neurons respond to auditory stimuli}

In a group of C57BL/6J mice $(n=9)$, we injected an AAV with a CaMKII promoter to express GCaMP6f (Chen et al., 2013) in the PPT on one side of the brain, and monitored neural activity as a function of calcium signals imaged with fiber photometry. The injection site is the location where bilateral optogenetic inhibition of CaMKII PPT neurons, or excitation of GABAergic synapses, abolishes signaled active avoidance (Hormigo et al., 2019). GCaMP6f expression was observed in a dorsoventral strip of cells, which included PPT, spanning from the ventral/posterior inferior colliculus to the dorsal/posterior substantia nigra (Fig. $1 A, B)$. Subsequently, a single optical fiber was implanted in the injection site using the same approach $\left(20^{\circ}\right.$ angle in the posterior direction) as the injection cannula. The optical fiber is estimated to image a volume $\left(2.5 \times 107 \mu \mathrm{m}^{3}\right)$ that extends $\sim 200 \mu \mathrm{m}$ from its ending (Pisanello et al., 2019; Pisano et al., 2019). We reconstructed in $3 \mathrm{D}$ the estimated imaging areas based on the optical fiber endings (Fig. 2). This imaging area covers a network of GCaMP6f-expressing neurons within the dorsal and middle parts of PPT (Fig. 1A, bottom panels). If the imaging extended further than the estimated area, it may include cells surrounding the dorsal PPT, such as the parabrachial and cuneiform nuclei. The mice underwent auditory mapping sessions, signaled active avoidance sessions, and unsignaled escape sessions while we measured calcium fluorescence signals $\left(\mathrm{F} / \mathrm{F}_{\mathrm{o}}\right)$ from $\mathrm{PPT}$ neurons.

During auditory mapping sessions (177 sessions in 7 mice), mice were placed in a small cage (half the size of a shuttle box) and eight auditory tones of different saliency were presented in random order ( $1 \mathrm{~s}$ tones every $4 \mathrm{~s}$, each repeated 10 times). We choose these tones because we have previously used them as CS in signaled active avoidance procedures. The calcium signal evoked in PPT neurons by the tones were dependent on the tone saliency (defined by sound pressure level [SPL] in dB; Fig. $1 C, D$, top); stronger calcium signals were evoked by higher-intensity tones. For instance, the $32 \mathrm{kHz}$ tone delivered at $57 \mathrm{~dB}$, which is around the baseline SPL ( $55 \mathrm{~dB})$, produced a negligible calcium signal; while a $4 \mathrm{kHz}$ tone delivered at $92 \mathrm{~dB}$ produced a very strong calcium signal. Reducing the saliency of the tone by lowering the intensity ( $4 \mathrm{kHz}$ from 92 to $68 \mathrm{~dB}$ or $8 \mathrm{kHz}$ from 81 to $66 \mathrm{~dB}$ ) lead to strong reductions in the evoked calcium signal. We simultaneously tracked movement using a color marker on the head implant. The more salient tones evoked an orienting movement that was noticeable as a small change (5\%-10\%) in speed versus the ongoing movement (Fig. 1C,D, bottom). The results show that PPT neurons respond robustly to salient auditory tones.

As a supplement, in a small group of mice ( 9 sessions in 3 mice), we tested the ability of six of the auditory tones to serve as a CS in a signaled active avoidance task in a shuttle box. In each trial of this task, an auditory CS predicts the US (foot-shock plus white noise) during a $7 \mathrm{~s}$ avoidance interval. If the animal shuttles between cage compartments during this period, it avoids the US (avoid). Otherwise, the US presentation drives the animal to shuttle (escape). Avoid or escape responses are followed by a variable ITI during which mice are free to shuttle (AA1 

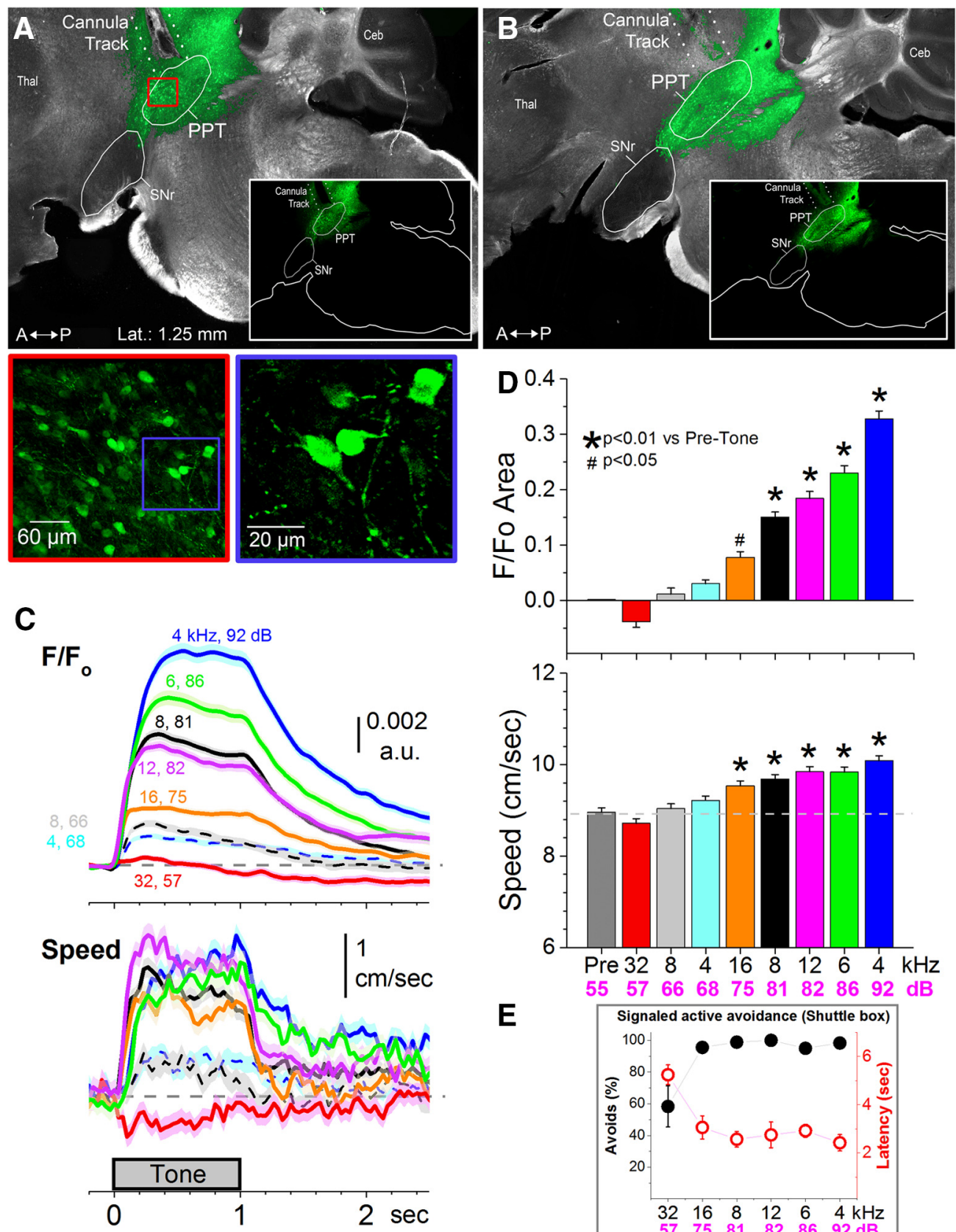

E

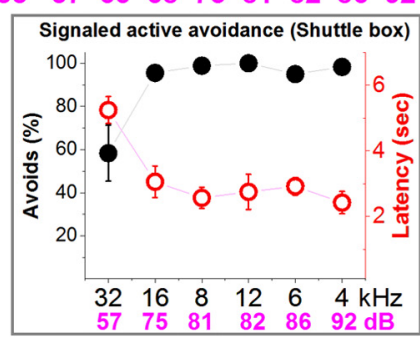

Figure 1. Effect of auditory tones on F/F calcium signals measured from PPT neurons using fiber photometry. $A$, Parasagittal section showing the optical fiber tract reaching PPT and GCaMP6f fluorescence around the fiber ending expressed in CaMKII neurons. The main panel blends a dark-field image of the section with the green channel of the GCaMP6f fluorescent image. Inset, The fluorescent image alone without blending. Bottom 2 panels, Close-ups of the GCaMP6f-labeled neurons in an area imaged by the optical fiber. Ceb, Cerebellum; Thal, thalamus; SNr, substantia nigra pars reticulata. $\boldsymbol{B}$, Same as in $\boldsymbol{A}$, but from another animal. $\boldsymbol{C}$, F/F $\mathrm{F}_{0}$ calcium imaging signals evoked from PPT neurons by auditory tones (1 s) of different saliency. The tones vary in frequency $(\mathrm{kHz})$ and SPL (dB). Bottom, Movement speed obtained by tracking a color marker on the head of the animal. The traces are mean \pm SEM of the auditory mapping sessions (177 audio sessions from 7 mice). $D$, Area of $F / F_{0}$ and speed measured during a time window $(0-3 \mathrm{~s})$ after tone onset. Significant Tukey test values comparing $F / F_{0}$ for each tone versus pretone (baseline): $16 \mathrm{kHz}, 75 \mathrm{~dB} t_{(2544)}=6.1, p<0.0001 ; 8 \mathrm{kHz}, 81 \mathrm{~dB} t_{(2544)}=14.0, p<0.0001 ; 12 \mathrm{kHz}, 82 \mathrm{~dB} t_{(2544)}=13.9, p<0.0001 ; 6 \mathrm{kHz}, 86 \mathrm{~dB} t_{(2544)}=16.0, p<0.0001 ; 4 \mathrm{kHz}$, $92 \mathrm{~dB} t_{(2544)}=22.0, p<0.0001$. Significant Tukey tests comparing speed for each tone versus pre-tone (baseline): $16 \mathrm{kHz}, 75 \mathrm{~dB} t_{(2544)}=4.4, p=0.048 ; 8 \mathrm{kHz}, 81 \mathrm{~dB} t_{(2544)}=5.8, p=$ $0.0013 ; 12 \mathrm{kHz}, 82 \mathrm{~dB} t_{(2544)}=6.5, p=0.0001 ; 6 \mathrm{kHz}, 86 \mathrm{~dB} t_{(2544)}=6.6, p<0.0001 ; 4 \mathrm{kHz}, 92 \mathrm{~dB} t_{(2544)}=8.8, p<0.0001$. E, Percentage of avoidance responses and response latencies for animals performing signaled active avoidance (AA1 procedure) using six of the auditory tones tested during the auditory mapping sessions in $\mathbf{C}$ and $\mathbf{D}$ as a CS. The low saliency tone (32 kHz, $57 \mathrm{~dB}$ ) was less effective at driving avoids compared with the other five more salient tones (9 sessions from 3 mice).

procedure). In this case, six different CS were randomly presented to signal the avoidance interval (Fig. 1E). Mice learned to avoid at high rates to each of the five salient CS, which evoked large calcium signals in PPT neurons during the auditory mapping sessions (Fig. 1C,D). In contrast, the low saliency tone $(32 \mathrm{kHz}$ at $57 \mathrm{~dB})$, which produced negligible calcium signals in
PPT neurons during the auditory mapping sessions, was not a very effective CS to drive active avoidance responses. These results suggest a relation between the ability to perform signaled active avoidance and CS-evoked PPT neuron activation. To directly test this relationship, we measured the activity of PPT neurons during signaled active avoidance. 


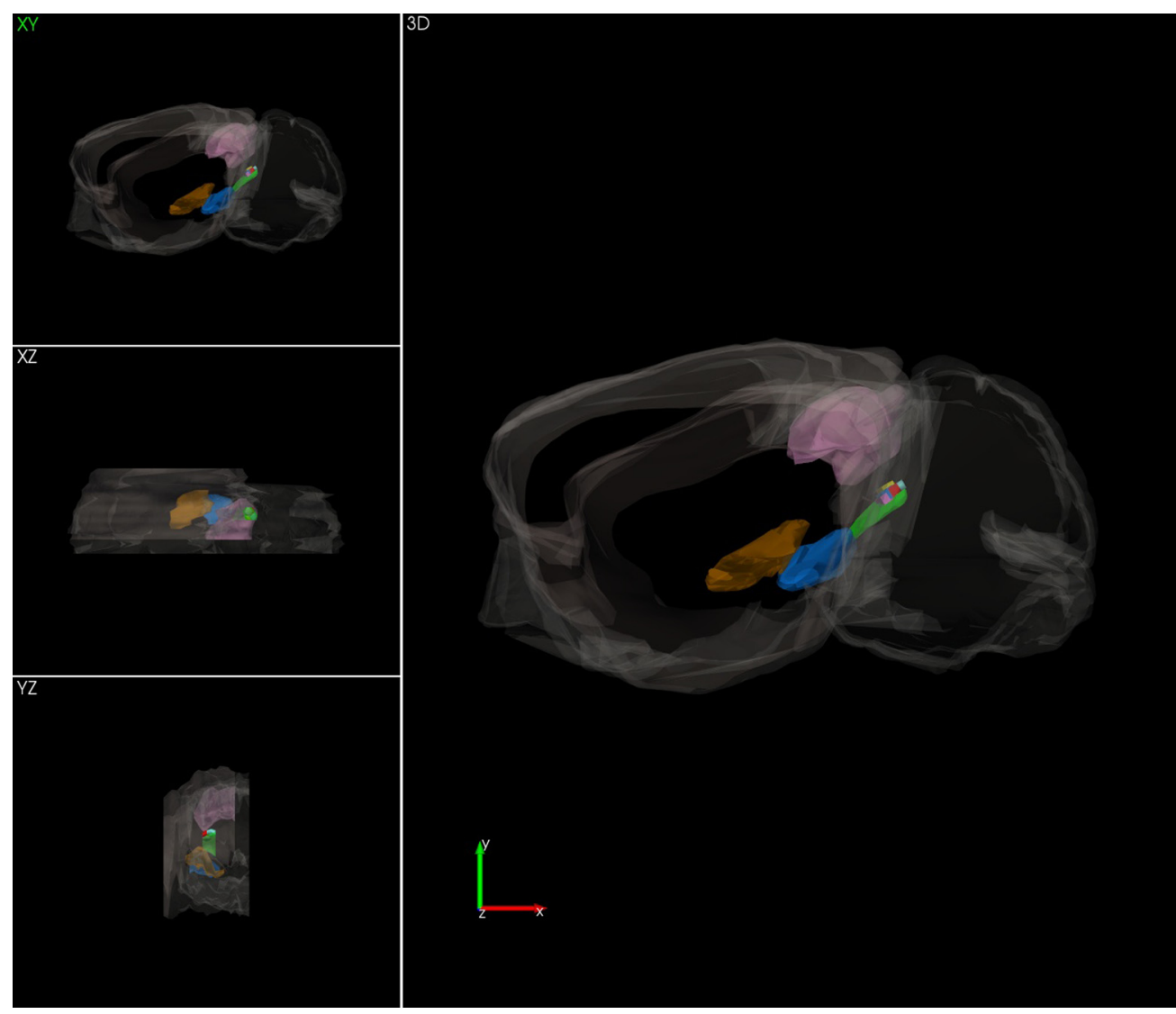

Figure 2. 3D reconstruction showing the PPT areas imaged by fiber photometry. The PPT region is the volume in semitransparent green. The small bright volumes (red, yellow, blue, pink, cyan) around the dorsal and middle portions of PPT represent the areas imaged per animal. This was traced by identifying the location of the optical fiber endings in parasagittal histologic sections and traced the estimated imaging area protruding from the cannula ending. Semitransparent pink represents the superior colliculus (dorsal to PPT). Semitransparent blue represents the $\mathrm{SNr}$ (rostral to PPT). Semitransparent orange represents the zona incerta (rostral to SNr). Figure 2 is a placeholder for Movie 1.

\section{PPT neurons robustly code signaled active avoidance}

We measured calcium signals in PPT neurons as mice performed signaled active avoidance in a shuttle box ( 35 sessions in 5 mice, AA1 procedure). The calcium signals plotted from CS trial onset revealed three noticeable peaks (Fig. $3 A$, dashed black trace; movement speed is shown in the bottom panels). The onset of the $\mathrm{CS}(8 \mathrm{kHz}$ at $81 \mathrm{~dB})$ during the avoidance interval evoked a sharp calcium peak (Peak 1 ) that was followed by a second much slower peak (Peak 2) a few seconds later. A third sharp peak (Peak 3) occurred during the onset of the escape interval (7 s from trial onset), when the US is presented (if the animals failed to avoid).

Separating the trials according to the response of the animals (avoids vs escapes; blue vs dashed red traces in Fig. 3 from CS onset) revealed that Peak 1 is evoked by the CS in either avoid or escape trials, regardless of the response of the animals. Moreover, Peak 1 is not associated with the movement to avoid since it occurs well before initiation of the avoidance movement. Peak 2 is associated with the avoidance response since it is prominent in avoid trials and concomitant with avoidance speed. Peak 3 is absent in avoid trials and is associated with the escape movement in escape trials.

The latency of avoidance responses driven by the CS during the avoidance interval is longer and more variable $(\sim 3.3 \pm 0.06 \mathrm{~s})$ than the latency of escape responses driven by the US during the escape interval $(0.5 \pm 0.02 \mathrm{~s})$; that is, mice respond very rapidly to the US and slowly to the CS. Thus, to determine whether Peak 2 is associated with avoid responses, we aligned the calcium signal with the occurrence of avoid or escape responses (Fig. 3, from response). This revealed a sharp calcium peak in association with the avoid responses, which was larger than the calcium peak evoked by the onset of the CS, but smaller than the calcium peak evoked by the faster escape responses. Thus, when the calcium signal is aligned by the occurrence of avoids, Peak 2 is fully revealed, and it is stronger than Peak 1 evoked by CS onset. Comparison of the calcium peak amplitude, time to peak, and area revealed that Peak 1 did not differ (Fig. 3D) between trials that produced avoids or escape responses (0-1 s window from CS onset). Peak 2 occurred only for avoids (2-7 s window from CS onset); there was no obvious Peak 2 in escapes, but the calcium signal remained elevated because of the Peak 1 driven by the CS and could rise further as mice began to avoid (but failed to make an avoid before US onset). Peak 3 was very large in amplitude and area for escapes, but absent for avoids (7-10 s window from CS onset).

During the ITI of the AA1 procedure, mice are free to shuttle and produce intertrial crossings. While avoids, escapes, or intertrial crossings involve the same overt shuttling response, they differ sharply in speed; the intertrial crossings occur on average at much slower speeds than avoids, and avoids occur at about half the speed of escapes (Fig. 3, green). Aligning the calcium signal with the occurrence of the responses revealed significant neural activity in PPT neurons during intertrial crossings, but this neural activity was virtually negligible compared with the neural activity associated with avoids and escapes. Thus, comparison of the area of the calcium signal between avoid, escape, and 


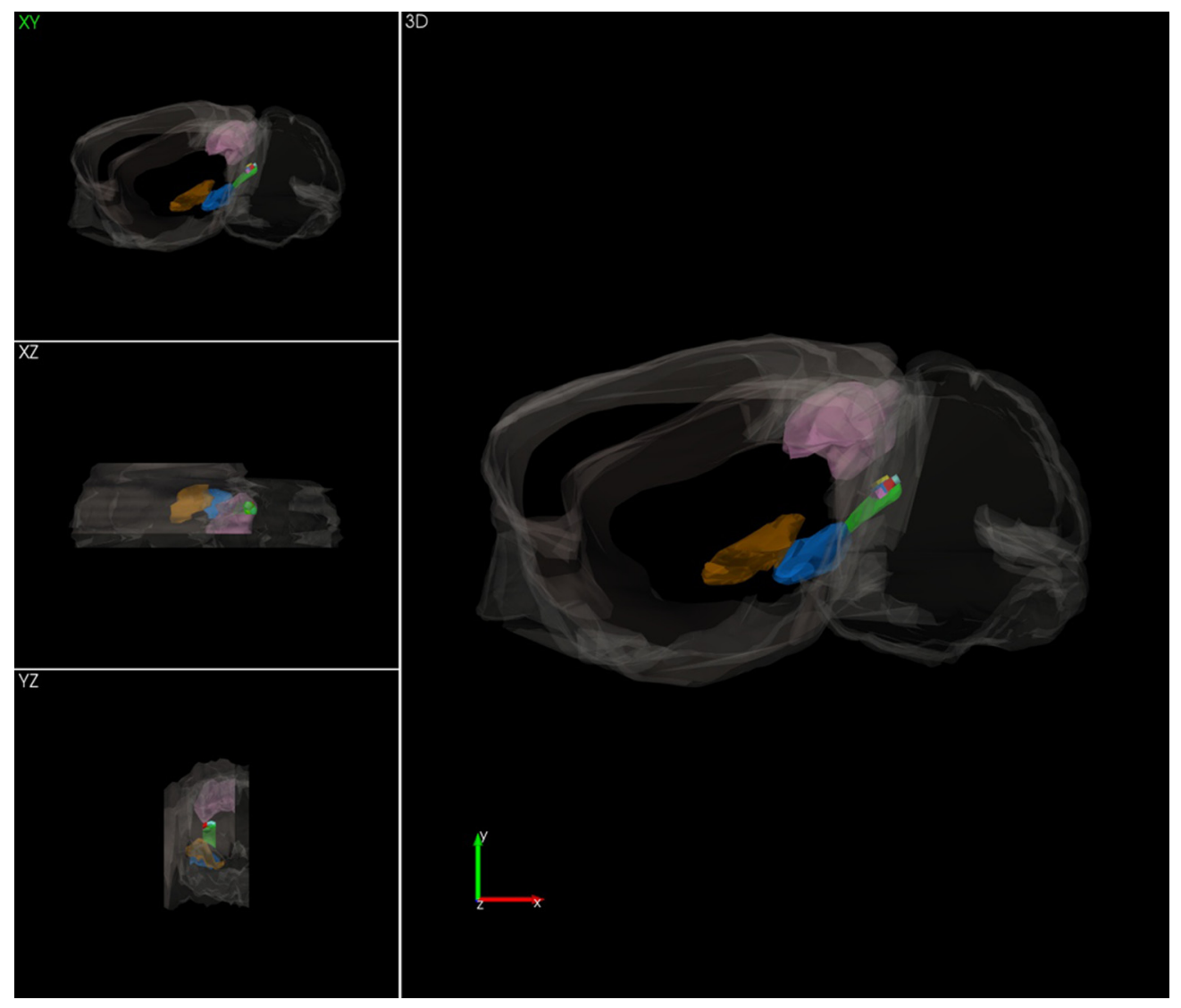

Movie 1. Video of the 3D reconstruction in Figure 2. [View online]

intertrial crossing responses (Fig. $3 D ;-1$ to $1 \mathrm{~s}$ window from response) revealed that avoids driven by the CS produced much stronger PPT neuron activation than spontaneous intertrial crossings, and the strongest PPT neuron activation occurred during the faster escape responses driven by the US.

Since these animals have high rates of avoidance responses, and consequently relatively few escape responses, we conducted additional sessions (Fig. 4; 32 sessions in 7 mice) in which the US (foot-shock and white noise) was presented unsignaled. The unsignaled US drives escape responses on every trial. To distinguish the contribution of white noise in the US, additional trials in the same session presented the foot-shock alone, without white noise. The unsignaled US evoked a strong calcium peak that was similar to Peak 3 evoked by the US in signaled active avoidance trials; thus, this peak was much larger than Peak 1 evoked by the CS (for comparison from the same animals, see Fig. $4 A$, gray trace). Removal of the white noise from the unsignaled US reduced the peak amplitude (not the area) of the calcium signal and increased the time to peak. The unsignaled US evoked fast escape responses that had faster peak speeds and escape latencies when the white noise was included (compared with the foot-shock alone), but the integrated movement speed or time to peak speed was not affected by inclusion of the white noise (Fig. $4 B$ ). Thus, in unsignaled US trials, inclusion of white noise enhances both the sharpness of the calcium peak signal in PPT neurons and the rapidity with which mice escape.

\section{Speed strongly correlates with calcium signals in PPT cells during signaled active avoidance}

We conducted a cross-correlation analysis between the movement speed and the calcium signal of PPT neurons during the signaled active avoidance sessions and the unsignaled US sessions. In general, speed and the calcium signal were strongly positively correlated. We compared the cross-correlations during three different periods of the signaled active avoidance task: the trial period (0-12 s from CS onset) separated according to the response of the animal (avoid vs escape), and an equivalent $12 \mathrm{~s}$ period during the ITI (Fig. $5 \mathrm{~A}$, left). This revealed strong crosscorrelations between speed and calcium signals that were strongest during trials leading to escape responses, followed by trials leading to avoid responses, and much weaker during the ITI (shuffled cross-correlations were virtually flat and not significant, $p>0.05$; data not shown). The cross-correlation for avoids had a prominent left-tail for the negative time periods (left side of the cross-correlogram) that spanned $>1 \mathrm{~s}$ and was absent for escapes. This likely reflects the fact that avoid responses are slower than escape responses, and possibly the presence of the calcium signal triggered by the CS (Peak 1 in Fig. 3), which precedes avoid responses. Comparison of the peak amplitude and time to peak of the cross-correlations (Fig. $5 B$, left) revealed that the calcium signal lagged the speed by $\sim 40 \mathrm{~ms}$ during avoids and escapes, and approximately double than that for movement during the ITI. A similar analysis for unsignaled US sessions revealed stronger cross-correlations during the escape responses compared with the ITI period (Fig. $5 A, B$, right). The cross-correlations for the escape responses evoked by the unsignaled US showed very little lag between speed and calcium signal, indicating that these variables are more closely related during unsignaled escapes.

We also performed a linear regression analysis between the speed and calcium signal (100 ms bins) for the same periods used for cross-correlations during signaled active avoidance (Fig. 5C). 

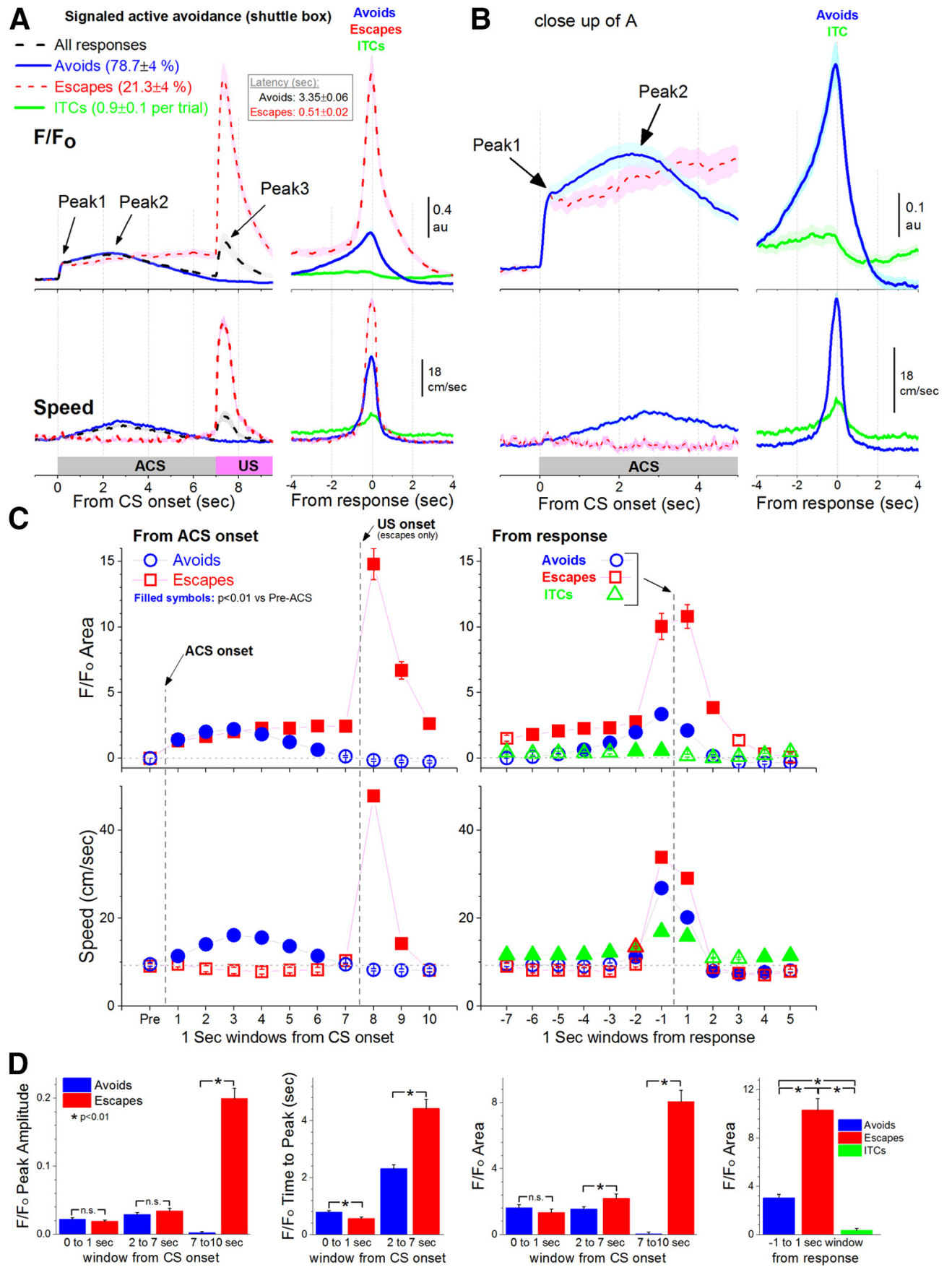

Figure 3. Effect of signaled active avoidance on F/F $F_{0}$ calcium signals measured from PPT neurons. $A, F / F_{0}$ calcium and speed traces (mean \pm SEM) from signaled active avoidance (AA1 procedure) sessions (35 sessions from 5 mice). At time 0 , traces are aligned from the CS onset (left panels) or from the response occurrence (right panels). The traces include all trials averaged together per session (dashed black) or separated according to the animal's response in the trial: avoids (blue) and escapes (dashed red). Also shown are the intertrial crossings (ITCs; produced during the ITI) aligned from response occurrence (green). The CS and US terminate when the mice avoid or escape, respectively; the mean \pm SEM avoid and escape response latencies (from CS or US onset, respectively) are noted. $\boldsymbol{B}$, Close-up of the traces in $\boldsymbol{A}$. Peak 1 in the $\mathrm{F} / \mathrm{F}_{0}$ calcium signal evoked by the $\mathrm{CS}$ for both avoids and escapes occurs before the onset of response movement (speed). Peak 2 in the $\mathrm{F} /$ $F_{0}$ calcium signal is associated with a rise in speed during avoidance responses and is fully revealed when the trace is aligned from the avoidance response occurrence (right panels). This $F / F_{0}$ Peak 2 is virtually inexistent during ITC, which involve the same movement as avoidance responses but performed more slowly. $C$, Area of $F / F_{0}$ and speed measured in $1 \mathrm{~s}$ windows from $C S$ onset (left) and from response occurrence (right) for avoids, escapes, and ITCS. Filled symbols represent a significant difference (Tukey test $p<0.01$ ) compared with a pretrial window shown on the left (Pre). D, The left 3 panels compare $\mathrm{F} / \mathrm{F}_{0}$ peak amplitude, time to peak, and area between avoids and escapes for 3 windows from CS onset (0-1, 2-7, and 7-10 s). Right, Comparison of $F / F_{0}$ area between avoids, escapes, and ITCS for a window around response occurrence $(-1$ to $1 \mathrm{~s})$. Significant Tukey test values comparing $F / F_{0}$ (from $C S$ onset) between avoids versus escapes: Peak Amplitude $(7-10 \mathrm{~s}), t_{(20)}=57.24$, $p<0.0001$; Time to Peak (0-1 s), $t_{(20)}=4.46, p=0.0049$; Time to Peak (2-7 s), $t_{(20)}=9.38, p<0.0001$; Area (2-7 s), $t_{(20)}=3.75, p=0.015$; Area $(7-10 \mathrm{~s}), t_{(20)}=35.17, p<0.0001$. Significant Tukey test values comparing $\mathrm{F} / \mathrm{F}_{0}$ area (from response): $t_{(38)}=28.33, p<0.0001$ (avoids vs escapes); $t_{(38)}=10.20, p<0.0001$ (avoids vs IT(S); $t_{(38)}=38.54, p<0.0001$ (escapes vs ITCS).

This revealed strong $r^{2}$ values that were significant $(p<0.01)$ for all sessions during all three periods. Moreover, when the calcium signal was shuffled, none of the sessions showed a significant linear regression (Fig. $5 C$, right). The $r^{2}$ values were much larger for avoids and escapes compared with the ITI period, indicating that the calcium signal is more linearly related to speed during the faster avoid and escape responses than during the slower ITI movement. 
A
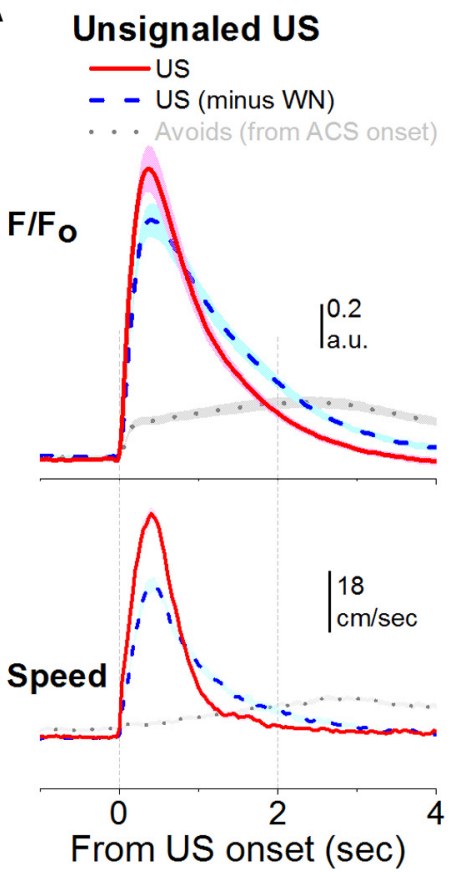

B $\square$ us (minus WN)
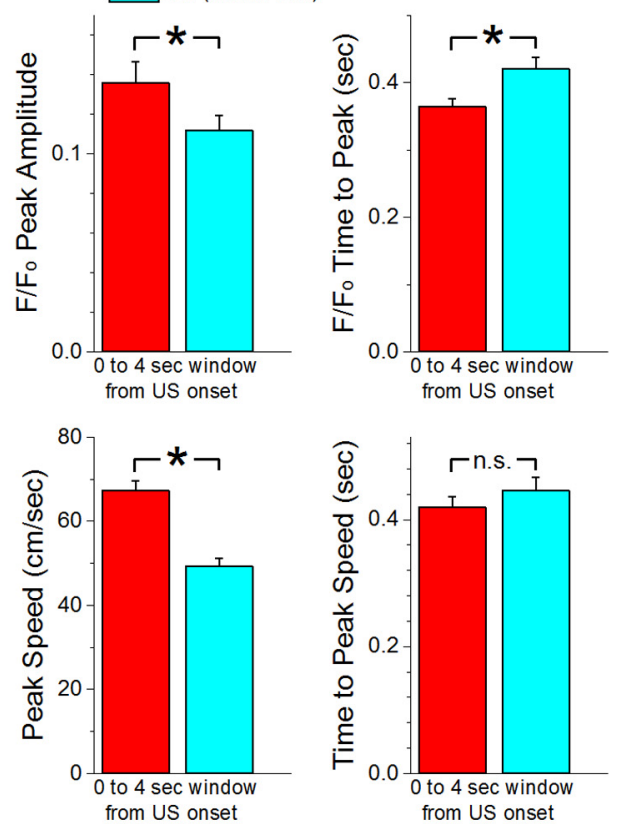
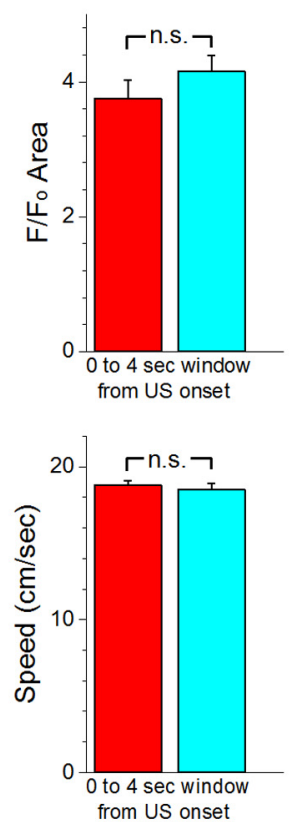

Figure 4. Effect of unsignaled US presentations on $\mathrm{F} / \mathrm{F}_{0}$ calcium signals measured from PPT neurons. $\boldsymbol{A}, \mathrm{F} / \mathrm{F}_{0}$ calcium and speed traces (mean $\pm \mathrm{SEM}$ ) from all unsignaled US sessions ( 32 sessions from 7 mice). At time 0, traces are aligned from US onset. Shown are trials evoked by the US (red) or the US without white noise (US-WN, dashed blue). Also shown for comparison is the trace evoked by the $\mathrm{CS}$ in signaled active avoidance (avoid) trials from Figure $3 A$ (for the same animals). $\boldsymbol{B}, \mathrm{F} / \mathrm{F}_{0}$ and speed peak amplitude, time to peak, and area measured during a $0-4 \mathrm{~s}$ window from US onset. The measures compare the effect of the US with (red) and without (cyan) white noise. Also shown is the latency of the escape response (bottom right). Significant Tukey test values comparing $F / F_{0}$ between US versus US-WN: Peak Amplitude, $t_{(27)}=9.7, p<0.0001$; Time to Peak (seconds), $t_{(27)}=5.3, p=0.0008$; Significant Tukey test values comparing speed between US versus US-WN: Peak Speed, $t_{(27)}=14.1, p<0.0001$; Latency, $t_{(27)}=14.0, p<0.0001$. $*$ significant at the levels indicated in the legend. n.S., not significant.

In addition, to determine whether the calcium signal at CS onset was reflecting different neural processing than the calcium signal at the end of the CS period (when animals avoid), we compared the cross-correlations between the initial $2 \mathrm{~s}$ after CS onset and the last $2 \mathrm{~s}$ before CS termination when animals avoid (for the same avoidance trials). The results revealed that the crosscorrelation between the calcium signals and speed was much stronger during the $2 \mathrm{~s}$ around avoidance than during the $2 \mathrm{~s}$ after CS onset (Tukey $t_{(29)}=14.8, p<0.0001$; cross-correlation peak or area). Since the calcium signals were similarly strong during these two periods of the avoidance trials, these results suggest that the PPT neural activity during these two periods reflect different neural processes (e.g., sensory detection and decision-making at CS onset vs avoidance response generation at CS offset).

Together, these results show that PPT neurons respond robustly to the CS that signals the US during signaled active avoidance behavior. In addition, PPT neurons respond even more sharply during avoidance and escape responses, with larger calcium signals associated with faster speeds. Thus, PPT neural activity has a full representation of signaled active avoidance.

PPT calcium signals closely track active avoidance responses In order to determine the relation between calcium signals and avoidance responses, we trained animals in three additional tasks that change the manner in which animals avoid.

First, animals that were trained in the basic signaled active avoidance AA1 procedure (used in Figs. 3-5) were trained in the same task with the exception that intertrial crossing responses are punished (AA2 procedure; 35 sessions from 5 mice). During AA2, mice almost entirely stop producing intertrial crossings without impairment in their avoidance rate. Interestingly, we found that in AA2 they consistently shifted their avoidance response latencies to longer values and increased the speed at which they avoid compared with AA1. This increases the separation between the CS onset and the avoidance response, and sharpens the response speed during a trial. One interpretation is that mice become more cautious about when to shuttle, postponing their decision to avoid until they are certain it is the correct option, and at that instance respond with more urgency. We found that these changes are reflected in the calcium signals measured from PPT neurons. Figure $6 A$ shows the average traces for the same animals performing AA1 and AA2 (Fig. 7 shows behavioral measures for these sessions). From CS trial onset, both the speed and the calcium signals shift to the right in accordance with the increase in avoidance response latency. From response occurrence, both the peak speed and calcium signal during avoidance responses were larger for AA2 compared with AA1 procedures (Fig. 6B). Thus, the activity of PPT neurons closely tracks the changes in the avoidance response associated with the AA2 procedure.

Second, we trained mice (48 sessions from 6 mice) in a discriminative task (AA3 procedure) in which two different $\mathrm{CS}$ are presented randomly. CS1 $(8 \mathrm{kHz}$ at $81 \mathrm{~dB}$; this is the same CS used in AA1 and AA2) requires mice to shuttle during the avoidance interval to actively avoid the US (this is akin to AA1 and $\mathrm{AA} 2)$. In contrast, $\mathrm{CS} 2(4 \mathrm{kHz}$ at $68 \mathrm{~dB})$ requires the mice to not shuttle during the avoidance interval to passively avoid presentation of the US for $0.5 \mathrm{~s}$ (if they shuttle). In other words, AA3 measures signaled active avoidance responses to CS1 and signaled passive avoidance responses to CS2. Mice perform this task very effectively (Fig. 7). As shown in Figure 6C, the speed from trial onset during avoids driven by CS1 is much larger than the speed during passive avoids driven by CS2, which is virtually 
flat. Since there are no active responses to CS2 during AA3, only the traces for active avoids are plotted from the response occurrence in Figure $6 C$ (right). Interestingly, while the calcium signal evoked by CS1 was similar to the signal observed during AA1 and AA2 active avoidance responses, the calcium signal evoked by CS2 was virtually flat in correspondence with the absence of a change in speed during passive avoidance responses; there was only a small peak at the onset of CS2 presentation at the start of the trial consistent with the signal evoked in mapping sessions (Fig. 1C,D). These results show that passive avoidance is not associated with an increase in the calcium signal of PPT cells. We also confirmed that mice $(n=9)$ can use this CS2 $(4 \mathrm{kHz}$ at $68 \mathrm{~dB})$ very effectively to perform active avoidance, displaying active avoidance rates $>90 \%$ when this CS signals the active avoidance interval.

Third, we trained mice (25 sessions from 5 mice) in a signaled active avoidance task in which three different CS correspond to different durations of the avoidance interval (AA4 procedure). In AA4, CS1 $(8 \mathrm{kHz}$ at $81 \mathrm{~dB}), \mathrm{CS} 2(10 \mathrm{kHz}$ at $82 \mathrm{~dB})$, and CS3 $(12 \mathrm{kHz}$ at $82 \mathrm{~dB})$ were associated with 4,7 , and $15 \mathrm{~s}$ avoidance interval durations, respectively. In AA4, mice shift their avoidance response latencies, so that the CS that signals a shorter avoidance interval drives faster avoidance response latencies (Figs. 6E,F, 7). From trial onset, the calcium signal matched the speed of the avoidance response. Moreover, the calcium signal of the shortest avoidance interval (CS1) was associated with the sharpest increase in calcium from response occurrence during avoidance responses. However, the peak speed of the responses to the three CS did not differ. In other words, the mice adjust the time at which they avoid, but when they do avoid, they do it at about the same speed. Thus, PPT neuron activation reflects the speed but also the urgency of the avoidance response.

These results indicate that PPT neurons shift their activity in accordance with the timing and urgency of the active avoidance response and do not discharge during passive avoidance.

\section{CS processing in PPT is required for signaled active avoidance learning}

The previous results show that neural activity in PPT neurons robustly represents signaled active avoidance behavior, including sharp neural responses during presentation of the CS. Expression of signaled active avoidance in trained mice requires these PPT neurons because optogenetically inhibiting them
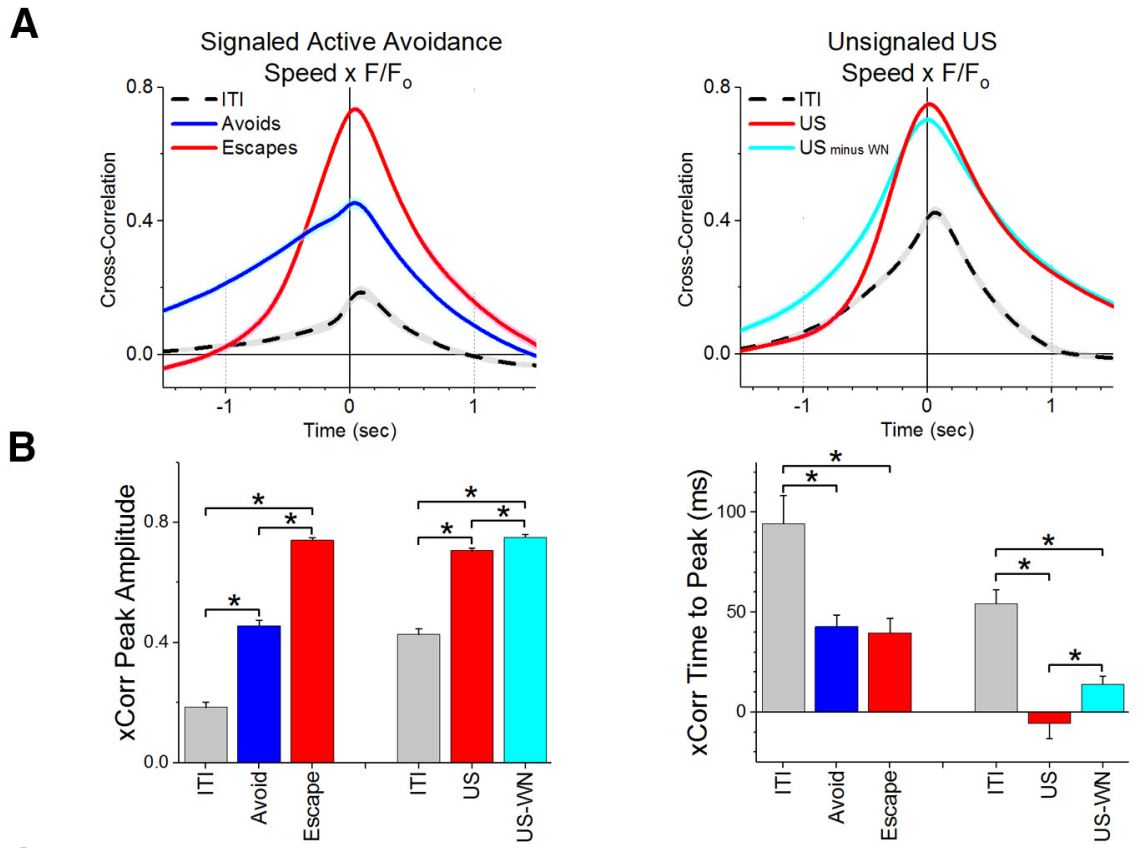

Signaled active avoidance
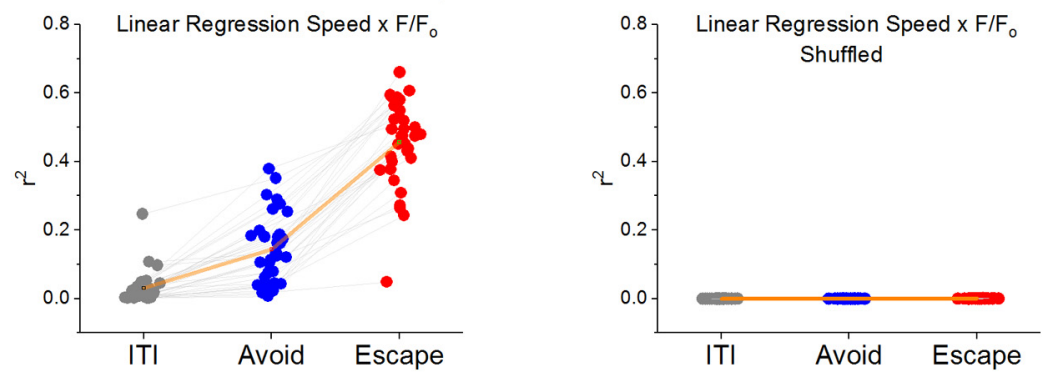

Figure 5. Cross-correlations between speed and $\mathrm{F} / \mathrm{F}_{0}$ calcium signals of PPT neurons during signaled active avoidance behavior and unsignaled US-evoked escape behavior. $A$, Cross-correlation analysis between speed and F/F calcium for signaled active avoidance (AA1 procedure, left) and unsignaled US sessions (right). In the signaled active avoidance sessions, the trial period (0-12 s from CS onset) was compared with the same amount of time during the ITI period ( -12 to $0 \mathrm{~s}$ preceding each trial period). Moreover, the cross-correlations during the trial period were computed separately for trials leading to avoids and escapes. For unsignaled US sessions, the same analysis was done comparing US presentations with or without white noise. All unsignaled US trials produce escape responses. $\boldsymbol{B}$, Peak amplitude and time to peak for the cross-correlations (xCorr) shown in $\boldsymbol{C}$. The time to peak denotes the lag between the speed and $\mathrm{F} / \mathrm{F}_{0}$ calcium signal. Significant Tukey test values for $x$ Corr Peak amplitude in signaled active avoidance: $t_{(58)}=23.3, p<0.0001$ (Avoids vs Escapes); $t_{(58)}=22.3148$, $p<0.0001$ (Avoids vs ITI); $t_{(58)}=45.61, p<0.0001$ (Escapes vs ITI). Significant Tukey test values for $x$ Corr Time to Peak in signaled active avoidance: $t_{(54)}=6.1, p=0.0002$ (Avoids vs ITI); $t_{(54)}=6.54, p<0.0001$ (Escapes vs ITI). Significant Tukey test values for $x$ Corr Peak Amplitude in Unsignaled US: $t_{(56)}=5.83, p=0.0003$ (US vs US-WN); $t_{(56)}=36.52, p<0.0001$ (US vs ITI); $t_{(56)}=42.36, p<0.0001$ (US-WN vs ITI). Significant Tukey test values for $x$ Corr Time to Peak in Unsignaled US: $t_{(56)}=4.65, p=0.0048$ (US vs US-WN); $t_{(56)}=14.19, p<0.0001$ (US vs ITI); $t_{(56)}=9.53, p<0.0001$ (US-WN vs ITI). $C$, Regression analysis between speed and $F / F_{0}$ during signaled active avoidance for the periods noted in $\boldsymbol{A}$. Plotted is the $r^{2}$ for the ITI, avoid, and escape periods of each session. Right, The same analysis after shuffling the $F / F_{0}$ signal. All $r^{2}$ values for the 3 periods in the left panel were statistically significant $(p<0.01)$, indicating a significant linear relation between speed and $\mathrm{F} / \mathrm{F}_{0}$ (none in the right panel was significant). This relation increases sharply during avoidance and, especially, escape movement compared with spontaneous ITI movement. *significant at the levels indicated in the legend. n.s., not significant.

during a trial, including both the avoidance and escape intervals, blocks avoidance responses to the CS without blocking escape responses to the US (Hormigo et al., 2019). In naive mice that express eArchT3.0 in PPT neurons (CaMKII-PPTArch mice; Fig. $8 A$ ), we explored whether inhibiting these PPT neurons with light only during the avoidance interval (ALCS trials) would affect the ability of the mice to learn the behavior. Under these conditions, PPT neurons would not be able to process the neural activity evoked by the CS or by emerging avoidance responses. 
A AA1 vs AA2 (punish ITCs)
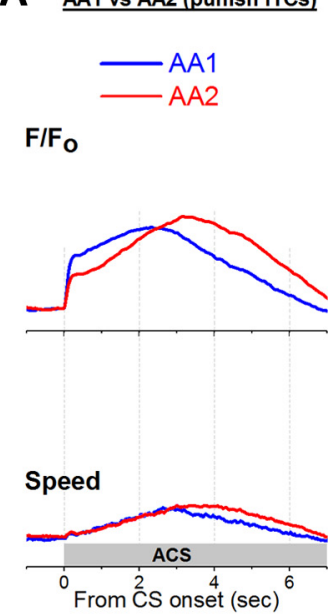

C

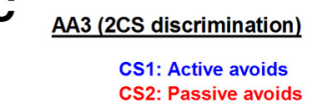

F/Fo
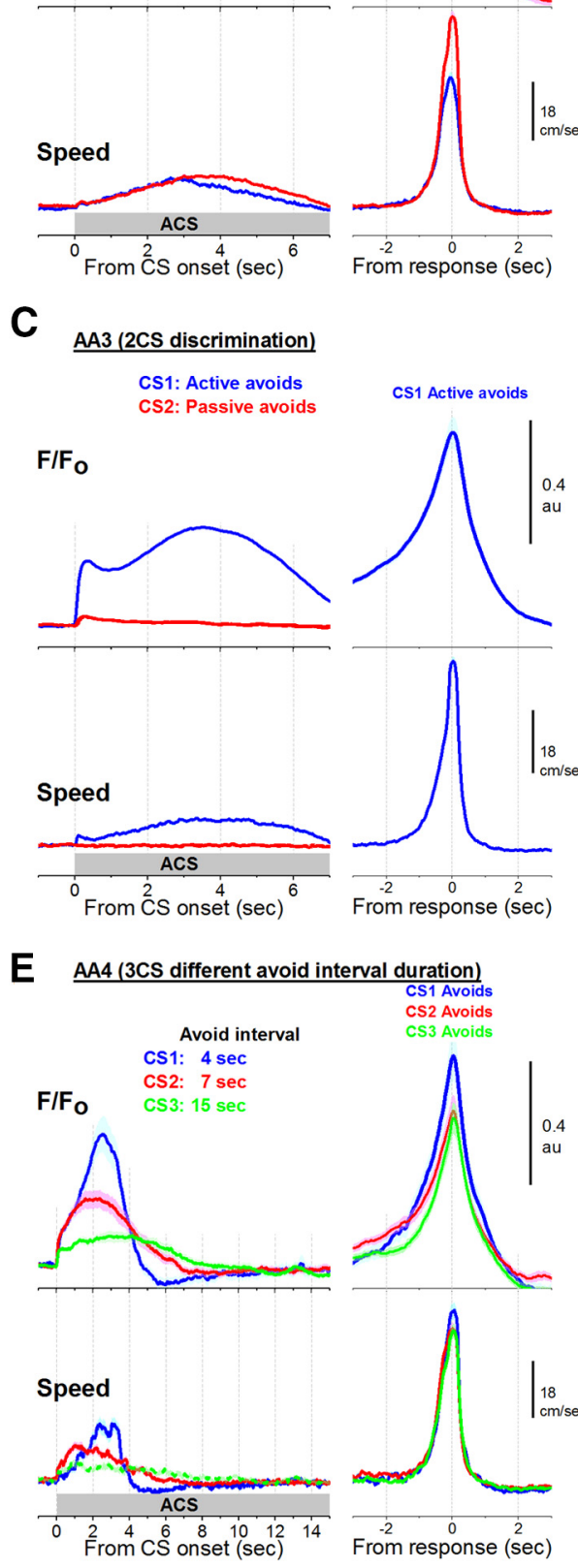

B
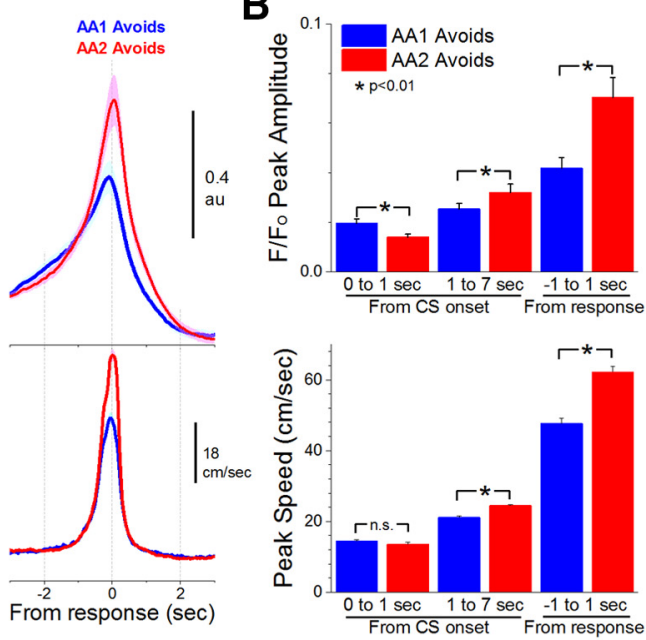

D
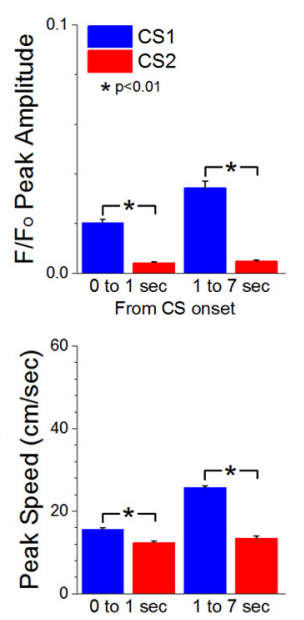

$\mathbf{F}$
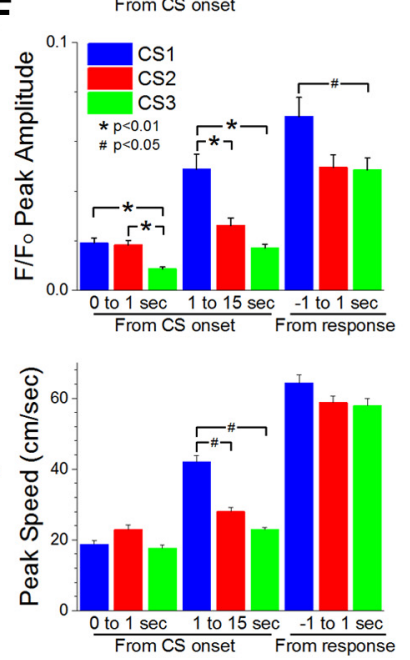
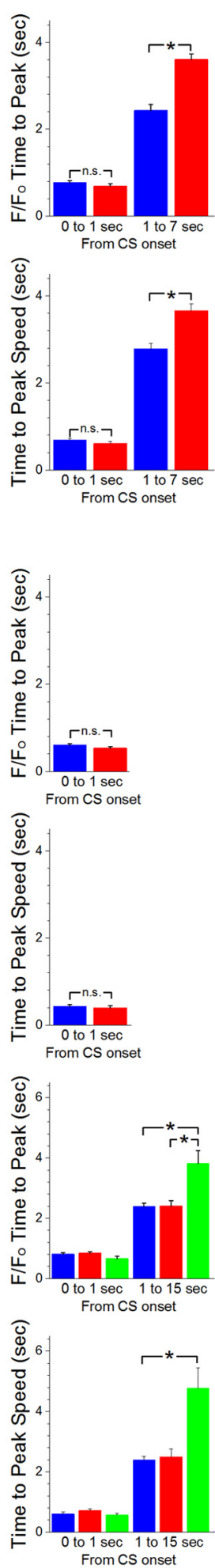

Figure 6. Effect of behavioral task contingency changes on avoidance responses and on $F / F_{0}$ calcium signals of PPT neuron. $A, F / F_{0}$ calcium and speed traces (mean $\pm S E M$ ) comparing avoidance responses from the same animals performing AA1 and AA2 signaled active avoidance sessions ( 35 sessions from 5 mice). In AA2, animals must passively avoid during the ITI. This task contingency change leads to longer latency active avoidance responses. The behavioral data for these sessions are shown in Figure 7. $B$, Top panels, Comparison of $F / F_{0}$ peak amplitude and time to peak for 2 windows from $C S$ onset $(0-1$ and 1-7 s) and 1 window around avoidance response occurrence $(-1$ to $1 \mathrm{~s})$ for avoidance responses during the AA1 and AA2 procedures. Bottom panels, Comparison of peak speed and time to peak speed for the same windows in the top panels. Significant Tukey test values comparing $F / F_{0}$ peak amplitude between AA1 versus AA2: $t_{(30)}=7.48, p<0.0001(0-1 \mathrm{~s}) ; t_{(30)}=4.56, p=0.003(1-7 \mathrm{~s}) ; t_{(30)}=11.82, p<0.0001$ ( -1 to $1 \mathrm{~s}$ ). Significant Tukey test values comparing F/F time to peak between AA1 versus AA2: $t_{(30)}=9.16, p<0.0001(1-7 \mathrm{~s})$. Significant Tukey test values comparing peak speed between AA1 versus AA2: $t_{(30)}=6.82, p<0.0001(1-7 \mathrm{~s}) ; t_{(30)}=12.19, p<0.0001(-1$ to $1 \mathrm{~s})$. Significant Tukey test values comparing time to peak speed between AA1 versus AA2: $t_{(30)}=6.56, p<0.0001$ (1-7 s). C, F/F $F_{0}$ calcium and speed traces (mean \pm SEM) comparing signaled active avoidance responses to CS1 and signaled passive avoidance responses to $C S 2$ for animals performing AA3 (48 sessions from 6 mice). In AA3, mice actively avoid during CS1 and passively avoid during CS2. The behavioral data for these sessions are shown in Figure 7. D. Top panels, Comparison of F/F peak amplitude and time to peak for 2 windows from $C S$ onset $(0-1$ and $1-7$ 
We found that naive CaMKII-PPT-Arch mice ( $n=5$; Fig. $8 B$, $C$, open black squares) trained with ALCS trials during two blocks of 5 sessions (50 trials/session) did not learn signaled active avoidance. The avoidance rate in the first block was $\sim 10 \%$. For comparison, a single block of 5 sessions in No Opsin controls subjected to the same optogenetic light (ALCS trials) show a $\sim 90 \%$ avoidance rate $(n=5$ mice; Fig. $8 B, C$, closed gray circles). The CaMKII-PPT-Arch mice received a test session (Test 1 ) consisting of CS presentations without US if they failed to avoid ( $n=25$ trials; Fig. $8 B, C$, open black squares) to test avoidance responses driven by the CS in the absence of further training. To test for spurious avoidance responses because of motor activity, these sessions also included randomly presented No CS trials in which the CS is not presented during the avoidance interval ( $n=25$ trials; Fig. $8 B, C$, closed red squares). The avoidance rate during the test session was very low $(\sim 20 \%)$ and not different compared with No CS trials, indicating that the CS had not acquired any ability to evoke active avoidance responses. Thus, although the CS predicts the US, it is unable to acquire the ability to evoke avoidance responses if PPT neurons are inhibited during the avoidance interval. Subsequent training of the CaMKII-PPT-Arch mice with regular CS trials without optogenetics (ACS trials) during two blocks of 5 sessions (50 trials/session) resulted in gradual learning of signaled active avoidance. A test conducted on the next day (Test 2) revealed large differences in avoidance rates between CS and No CS trials, indicating that the CS had acquired the ability to evoke avoidance responses during training without optogenetic light. These results indicate that $\mathrm{PPT}$ neurons must process the avoidance interval for the CS to drive avoidance responses (i.e., for effective signaled active avoidance learning to occur). Finally, we also show the effect of PPT neuron inhibition on the ability (speed) of animals to perform escape responses driven by the US. As shown in Figure $8 D$, the same optogenetic PPT inhibition that was effective at abolishing active avoidance to an ACS had negligible effects on the speed with which the same animals escape the US $(n=5$ mice, peak speed, Tukey $t_{(16)}=1.6, p=0.3$; Control vs Green Light in

\footnotetext{
s) for AA3 procedure active avoidance responses during $\mathrm{CS} 1$ and passive avoidance responses during CS2. Bottom panels, Comparison of peak speed and time to peak speed for the same windows as the top panels. Significant Tukey test values comparing $F / F_{0}$ Peak Amplitude between CS1 versus CS2: $t_{(42)}=24.59, p<0.0001(0-1 \mathrm{~s}) ; t_{(42)}=34.73, p<0.0001(1-7$ s). Significant Tukey test values comparing peak speed between CS1 versus CS2: $t_{(42)}=$ $10.41, p<0.0001(0-1 s) ; t_{(42)}=32.99, p<0.0001$ (1-7 s). $E, F / F_{0}$ calcium and speed traces (mean \pm SEM) comparing avoidance responses to CS1, CS2, and CS3 for animals performing AA4 (25 sessions from 5 mice). In AA4, the 3 distinct CS tones $(8,10$, and $12 \mathrm{kHz}$ at $\sim 80 \mathrm{~dB}$ ) signal different active avoidance interval durations of 4,7 , and $15 \mathrm{~s}$. Mice adjust their active avoidance response latency according to the duration of the signaled active avoidance interval. The behavioral data for these sessions is shown in Figure 7. $\boldsymbol{F}$, Top panels, Comparison of $F / F_{0}$ peak amplitude and time to peak for 2 windows from $C S$ onset (0-1 and $2-7 \mathrm{~s})$ and 1 window around response occurrence ( -1 to $1 \mathrm{~s}$ ) active avoidance responses during CS1, CS2, and CS3 (AA4 procedure). Bottom panels, Comparison of peak speed and time to peak speed for the same windows as the top panels. Significant Tukey test values for $\mathrm{F} / \mathrm{F}_{0}$ peak amplitude (0-1 s): $t_{(38)}=6.74, p<0.0001$ (CS1 vs $(S 3) ; t_{(38)}=5.55, p=0.001$ (CS2 vs CS3). Significant Tukey test values for $F / F_{0}$ peak amplitude (1-15 s): $t_{(38)}=5.84, p=$ 0.0005 (CS1 vs CS2); $t_{(38)}=8.69, p<0.0001$ (CS1 vs CS3). Significant Tukey test values for $\mathrm{F} / \mathrm{F}_{0}$ peak amplitude ( -1 to $1 \mathrm{~s}$ ): $t_{(38)}=4.11, p=0.0164$ (CS1 vs (S3). Significant Tukey test values for $\mathrm{F} / \mathrm{F}_{0}$ time to peak (1-15 s): $t_{(38)}=4.05, p=0.0179$ (CS1 vs $(\mathrm{S} 3) ; t_{(38)}=3.61$, $p=0.0381$ (CS2 vs CS3). Significant Tukey test values for peak speed (1-15 s): $t_{(38)}=8.29$, $p<0.0001$ (CS1 vs $(S 2) ; t_{(38)}=11.48, p<0.0001$ (CS1 vs CS3). Significant Tukey test values for time to peak speed (1-15 s): $t_{(38)}=4.51, p=0.0078$ (CS1 vs CS3). $*$ significant at the levels indicated in the legend. n.s., not significant.
}

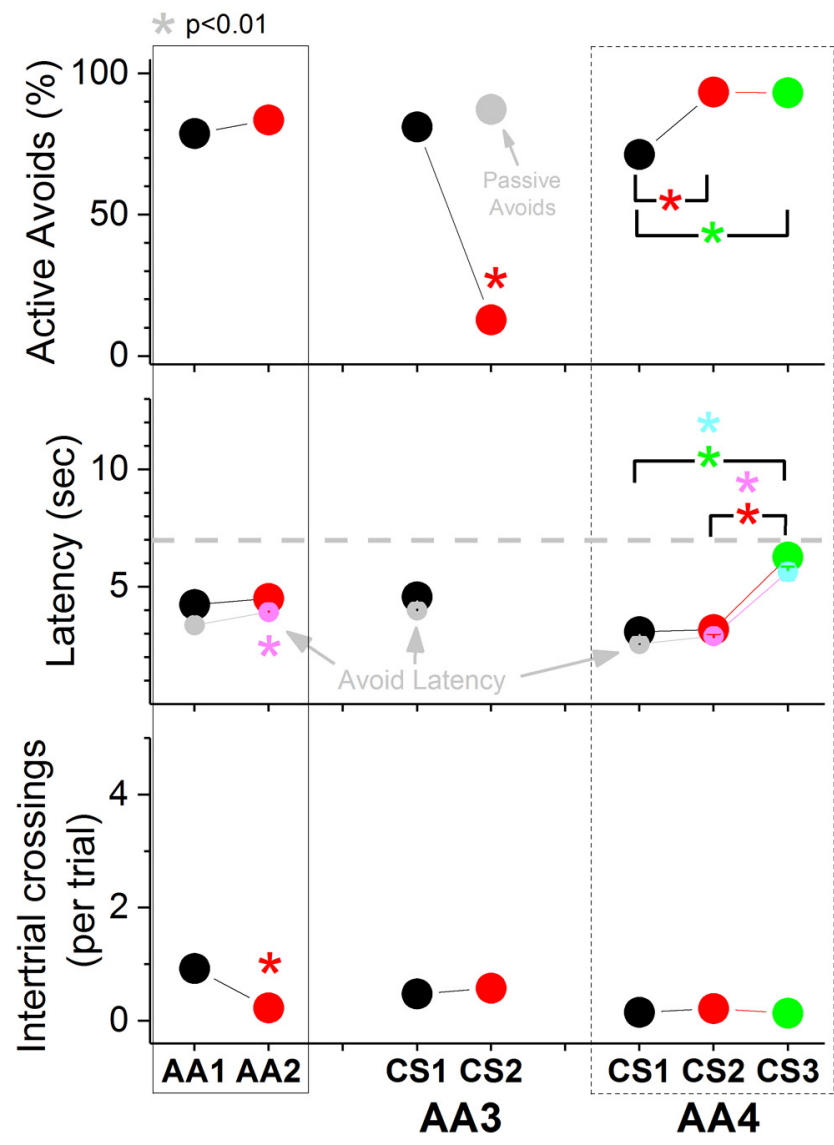

Figure 7. Behavioral performance during the different signaled active avoidance procedures. Percentage of active avoidance responses (top), response latency (middle), and intertrial crossings (bottom) for the different signaled active avoidance procedures shown in Figure 6 (AA1, $A A 2, A A 3$, and AA4). In $A A 3$, the correct response to $C S 2$ is a passive avoidance (top, shown in gray). The middle panel also shows in gray the avoidance latencies (i.e. response latency excluding escape latency). Significant Tukey test values for Active Avoids: $t_{(42)}=54.25, p<0.0001$ (CS1 vs $\left(\mathrm{CS} 2\right.$ in AA3); $t_{(38)}=5.27, p=0.0018$ (CS1 vs (S2 in AA4); $t_{(38)}=5.41, p=0.0013$ (CS1 vs (S3 in AA4). Significant Tukey test values for latency: $t_{(38)}=9.54, p<0.0001$ (CS1 vs $\left(S 3\right.$ in AA4); $t_{(38)}=8.63$, $p<0.0001$ (CS2 vs CS3 in AA4). Significant Tukey test values for avoid latency: $t_{(23)}=9.63, p<0.0001$ (AA1 vs AA2); $t_{(38)}=10.98 p<0.0001$ (CS1 vs (S3 in AA4); $t_{(38)}=9.21, p<0.0001$ (CS2 vs CS3 in AA4). Significant Tukey test values for intertrial crossings: $t_{(23)}=10.42, p<0.0001$ (AA1 vs AA2).

PPT of CaMKII-PPT-Arch mice). Thus, the level of PPT inhibition sufficient to block signaled active avoidance does not suppress escaping, as previously shown (Hormigo et al., 2019).

\section{Discussion}

Using calcium imaging fiber photometry in freely behaving mice performing signaled active avoidance, we found that PPT neurons in the midbrain locomotor region sharply activate during presentation of the auditory CS that predicts the threat before onset of the avoidance movement. PPT neurons activate further during the succeeding CS-driven avoidance movement or during the US-driven escape movement. PPT neuron activation was weak during spontaneous exploratory movements but correlated sharply with movement speed and, therefore, with the urgency of the response. Thus, neurons in the midbrain tegmentum process the CS that predicts the threat, and discharge during the occurrence of conditioned (avoidance) and unconditioned 
A

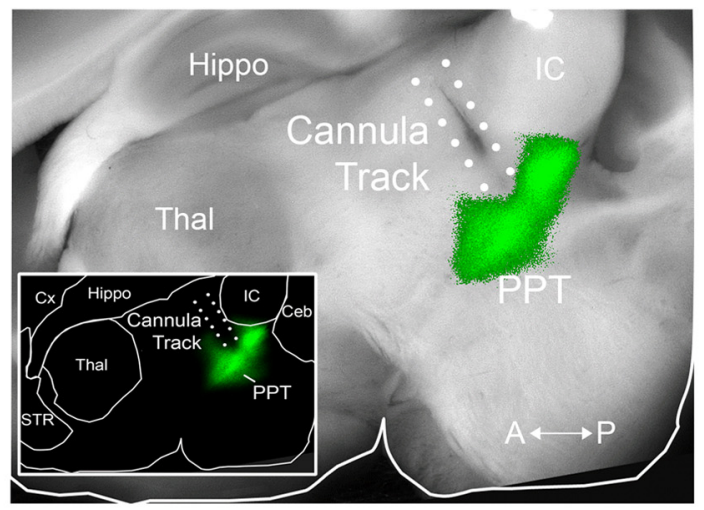

B
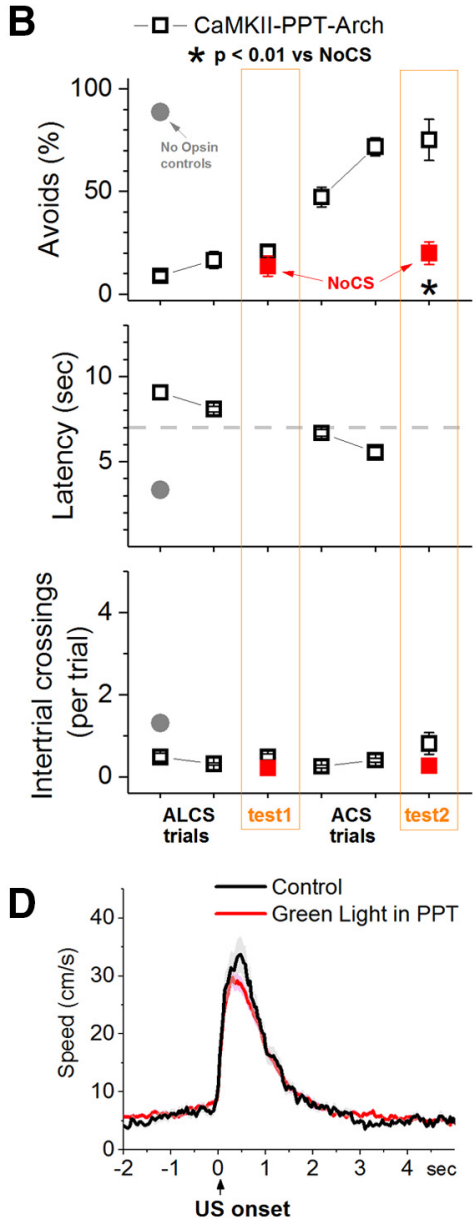

Figure 8. Effect of PPT neuron inactivation during the avoidance interval on signaled active avoidance learning. $\boldsymbol{A}$, Parasagittal section showing the optical fiber tract reaching PPT and eArchT3.0 expression around the fiber ending expressed in CaMKII neurons (CaMKII-PPT-Arch mice). The main panel blends a bright-field image of the section with the green channel of the eYFP fluorescent image. Inset, The fluorescent image alone without blending. $\mathrm{C}$, Cortex; Ceb, cerebellum; Hippo, hippocampus; Thal, thalamus; Str, striatum. $\boldsymbol{B}$, Percentage of avoidance responses, latency, and intertrial crossings for mice that express eArchT3.0 in PPT neurons (CaMKII-PPT-Arch; 5 mice). Plots represent 6 blocks of sessions. In the initial 2 blocks ( 5 sessions per block), naive mice perform ALCS trials during which optogenetic green light is delivered bilaterally to inhibit PPT neurons together with the CS during the avoidance interval (AA1 Procedure). The third block (Test 1) tests the ability of the mice to avoid during presentation of the CS without optogenetic light (the escape interval and US are not presented if the animal does not avoid). No CS trials are presented randomly for comparison (solid, red squares). In No CS trials, the CS is omitted during the avoidance interval to check for spurious avoids because of normal movement. The fourth and fifth blocks are identical to the first 2 blocks but without optogenetic light. The final block is a repeat of the test done in the third block. Gray filled circles in the first block represent a group of No Opsin mice (that do not express eArchT3.0; $n=4$ ) implanted with dual cannulas in PPT and subjected to the same optogenetic light protocol as the experimental mice. These mice learned the task in the first block indicating that the impairment in the experimental mice was caused by inhibiting PPT neurons, not by the light itself. Significant Tukey test values comparing $C S$ trials (without US) and № CS trials in the two test sessions: $t_{(4)}=5.6, p=0.009$ (Test 2). C, Trial speed, trial velocity, and intertrial speed for the data shown in $\boldsymbol{B}$. D, Overlay of speed for escape responses triggered by presentation of the US during the absence (control) or presence of green light in the PPT of CaMKII-PPT-Arch mice. Escape response speed is similar in both conditions. (escape) responses. Using optogenetics, we found that these neurons must discharge during the signaled avoidance interval for naive mice to effectively learn signaled active avoidance behavior. Together, the results point to the PPT area in the midbrain locomotor region as an essential hub for signaled active avoidance behavior.

\section{PPT neurons respond to auditory tones, including the CS}

The PPT is reciprocally connected with the basal ganglia, has ascending cholinergic projections to the thalamus and other forebrain areas, and has descending projections to the deep nuclei of the cerebellum and the medulla (Inglis and Winn, 1995; Mena-Segovia et al., 2004; Mori et al., 2016; Roseberry et al., 2016; Caggiano et al., 2018). We found that PPT neurons discharge at short latency to presentation of the CS during signaled active avoidance and discharge to auditory tones of different frequencies as a function of SPL; the calcium response increases as the stimulus becomes louder. Indeed, PPT neurons have been shown to discharge to auditory tones in cats and rats, and this activity can drive substantia nigra dopamine neurons through tegmentonigral connections (Reese et al., 1995; Pan and Hyland, 2005); indeed, PPT neuron activation can be reinforcing (Yoo et al., 2017). The source of the auditory afferents that drive the PPT neurons is uncertain but may include direct or indirect projections to the midbrain tegmentum from cochlear root neurons (López et al., 1999; Nodal and Lopez, 2003), from the cochlear nuclei (Kandler and Herbert, 1991), and/or from the auditory cortex (Schofield and Motts, 2009). In addition, neurons in the adjacent cuneiform nuclei, which are also part of the midbrain locomotor region, discharge to sensory stimuli via afferents from superior colliculus (Westby et al., 1990). Consistent with the auditory mapping sessions, PPT neurons discharged to the CS during signaled active avoidance sessions. Thus, PPT and the related locomotor region have direct access to auditory sensory inputs that can function as effective CS to learn behavioral contingencies.

\section{PPT neurons activate during} avoidance and escape responses In addition to discharging at the onset of the CS, PPT neurons discharged 
during both avoidance and escape responses during signaled active avoidance. Avoidance responses driven by the CS produced much stronger PPT neuron activation than spontaneous movement. Indeed, the level of PPT activation during shuttling in the ITI was almost negligible compared with the activation during avoidance responses. The largest level of PPT neuron activation occurred during escape responses driven by the US. However, escape responses also include the effect of the footshock and white noise. The white noise contributes to the sharpness of the calcium signal and the peak speed of the escape movement, but most of the effect is caused by the foot-shock. In general, PPT activation correlated strongly with speed and the apparent urgency of the response. The relevance of the urgency of the response was evident in the AA4 procedure, where mice adjust their avoidance response according to the duration of the avoidance interval (signaled by a distinct CS). In this procedure, mice produce avoidance responses of similar peak speed to the three CS in AA4, but the PPT activation was stronger for the more urgent response evoked by CS1, which signals a shorter (4 s) avoidance interval. Interestingly, using the AA3 procedure, we also found that signaled passive avoidance (CS2 in AA3) did not produce PPT activation. Thus, it seems that only signaled active avoidance, not signaled passive avoidance, is represented by neuronal activity in PPT.

Our findings are in good agreement with previous studies that have shown that PPT and midbrain locomotor region neurons discharge in relation to movement speed, which they control through descending projections to caudal brainstem (Garcia-Rill and Skinner, 1988; Roseberry et al., 2016; Capelli et al., 2017; Caggiano et al., 2018). Furthermore, we revealed an integrated activation of PPT neurons during signaled active avoidance that completely represents this behavior, including the conditioned cue and both the conditioned and unconditioned responses. Thus, consistent with an essential role as a hub for signaled active avoidance, PPT circuits have all the elements needed to perform this behavior. It is no surprise that inhibiting these neurons, or exciting GABAergic neurons, in PPT blocks the expression of active avoidance behavior (Hormigo et al., 2019). Moreover, the large difference in PPT neuronal activation between avoidance and escape responses likely explains why the same level of optogenetic inhibition blocks avoidance responses but not escape responses (Fig. $8 D$ ). One possibility is that the PPT is a common pathway for expression of both avoidance and escape responses, which differ in neuronal activation levels because of the distinct sensory afferents and, especially, intensity levels produced by CS and US. While our main focus is on the learned avoidance behavior, the dorsal periaqueductal gray in the midbrain has been shown to play an important role in producing escape responses (Bandler et al., 1985; Brandao et al., 1994), and afferents from this area may account for the US-driven escaperelated activity we observed in PPT.

An important consideration for future work regards the afferents that produce the CS-driven avoidance-related activity in PPT. One scenario is that this activity is driven by the cooperation between multiple areas, including the output of the basal ganglia, the zona incerta, the superior colliculus, and other nuclei, such as the amygdala and the neocortex, all of which have direct or indirect projections to this area. Indeed, the discharge of superior colliculus neurons during avoidance responses resembles the activity we observed in PPT (Cohen and CastroAlamancos, 2010b), but solely suppressing this activity does not impair signaled active avoidance (Cohen and Castro-Alamancos, 2007; Hormigo et al., 2019). Moreover, both the basal ganglia and zona incerta provide GABAergic afferents to PPT that can powerfully suppress active avoidance responses (Hormigo et al., 2016, 2020a). These GABAergic inputs may control (facilitate or impede) the ability of other afferents to drive avoidance responses in PPT (Hormigo et al., 2020b). Future work will focus on identifying the sources of the CS-driven avoidance-related activity that arises in PPT.

\section{PPT neuron activation is required for learning signaled active avoidance}

The finding that PPT neuronal activation is required for signaled active avoidance learning is intriguing. In this experiment, where we inhibited PPT neurons during the avoidance interval, the CS predicts the US, but mice appear to learn nothing about this relation. One reason learning does not develop is because mice fail to experience avoidance responses during these learning sessions (mice escape on every trial in response to the US but barely avoid). Thus, while the Pavlovian contiguity between the CS and US is present, the operant reinforcement contingency (consequence) of removing the CS (and US) during an avoidance response is not experienced at a sufficient rate to drive learning. In essence, our interpretation is that, by failing to experience the avoidance response, mice do not learn the behavior. Moreover, when the animals are tested without optogenetic stimulation (Test 1 ) they do not display any appreciable response to the CS, including freezing (trial speed is not different between CS and No CS trials in Test 1). Thus, mice may have also failed to acquire the Pavlovian fear association that is thought to be the first component of active avoidance learning.

\section{What is the role of PPT in signaled active avoidance?}

The results reinforce the notion that the PPT area is an essential hub for the acquisition and expression of signaled active avoidance and raise a few points. First, the rapid PPT neuronal activation at CS onset (Peak 1) driven by auditory afferents may serve as a wake-up call to prepare the PPT for a possible upcoming avoidance response. Second, the avoidance response signal observed in PPT (Peak 2) is likely driven by afferents that originate from a distributed network that makes the decision to avoid. An important future goal will be to determine the origin of the inputs that drive Peak 2. This may include direct or indirect projections to PPT from forebrain areas (e.g., amygdala, accumbens, frontal cortex) and/or midbrain areas (e.g., superior colliculus, substantia nigra) known to regulate avoidance. Finally, escape responses driven by the US are associated with a stronger PPT activation (Peak 3) than avoidance responses. The afferents recruited by the US that drive Peak 3 may originate in the same network as the afferents that drive Peak 2 or, more likely, in a different network.

\section{References}

Amorapanth P, LeDoux JE, Nader K (2000) Different lateral amygdala outputs mediate reactions and actions elicited by a fear-arousing stimulus. Nat Neurosci 3:74-79.

Arber S, Costa RM (2018) Connecting neuronal circuits for movement. Science 360:1403-1404.

Bandler R, Depaulis A, Vergnes M (1985) Identification of midbrain neurones mediating defensive behaviour in the rat by microinjections of excitatory amino acids. Behav Brain Res 15:107-119.

Blanchard DC, Blanchard RJ, Griebel G (2005) Defensive responses to predator threat in the rat and mouse. Curr Protoc Neurosci 8:19.

Bolles RC (1970) Species-specific defense reactions and avoidance learning. Psychol Rev 77:32-48. 
Branco T, Redgrave P (2020) The neural basis of escape behavior in vertebrates. Annu Rev Neurosci 43:417-439.

Brandao ML, Cardoso SH, Melo LL, Motta V, Coimbra NC (1994) Neural substrate of defensive behavior in the midbrain tectum. Neurosci Biobehav Rev 18:339-346.

Bravo-Rivera C, Roman-Ortiz C, Brignoni-Perez E, Sotres-Bayon F, Quirk GJ (2014) Neural structures mediating expression and extinction of platform-mediated avoidance. J Neurosci 34:9736-9742.

Caggiano V, Leiras R, Goni-Erro H, Masini D, Bellardita C, Bouvier J, Caldeira V, Fisone G, Kiehn O (2018) Midbrain circuits that set locomotor speed and gait selection. Nature 553:455-460.

Capelli P, Pivetta C, Soledad Esposito M, Arber S (2017) Locomotor speed control circuits in the caudal brainstem. Nature 551:373-377.

Chen TW, Wardill TJ, Sun Y, Pulver SR, Renninger SL, Baohan A, Schreiter ER, Kerr RA, Orger MB, Jayaraman V, Looger LL, Svoboda K, Kim DS (2013) Ultrasensitive fluorescent proteins for imaging neuronal activity. Nature 499:295-300.

Cohen JD, Castro-Alamancos MA (2007) Early sensory pathways for detection of fearful conditioned stimuli: tectal and thalamic relays. J Neurosci 27:7762-7776.

Cohen JD, Castro-Alamancos MA (2010a) Detection of low salience whisker stimuli requires synergy of tectal and thalamic sensory relays. J Neurosci 30:2245-2256

Cohen JD, Castro-Alamancos MA (2010b) Neural correlates of active avoidance behavior in superior colliculus. J Neurosci 30:8502-8511.

Garcia-Rill E, Skinner RD (1988) Modulation of rhythmic function in the posterior midbrain. Neuroscience 27:639-654.

Hersman S, Allen D, Hashimoto M, Brito SI, Anthony TE (2020) Stimulus salience determines defensive behaviors elicited by aversively conditioned serial compound auditory stimuli. Elife 9:e53803.

Hormigo S, Vega-Flores G, Castro-Alamancos MA (2016) Basal ganglia output controls active avoidance behavior. J Neurosci 36:10274-10284.

Hormigo S, Vega-Flores G, Rovira V, Castro-Alamancos MA (2019) Circuits that mediate expression of signaled active avoidance converge in the pedunculopontine tegmentum. J Neurosci 39:4576-4594.

Hormigo S, Zhou J, Castro-Alamancos MA (2020a) Zona incerta GABAergic output controls a signaled locomotor action in the midbrain tegmentum. eNeuro 7:ENEURO.0390-19.2020.

Hormigo S, Zhou J, Chabbert D, Shanmugasundaram B, Castro-Alamancos MA (2020b) Basal ganglia output has a permissive non-driving role in a signaled locomotor action mediated by the midbrain. J Neurosci 41:1529-1552.

Inglis WL, Winn P (1995) The pedunculopontine tegmental nucleus: where the striatum meets the reticular formation. Prog Neurobiol 47:1-29.

Kandler K, Herbert H (1991) Auditory projections from the cochlear nucleus to pontine and mesencephalic reticular nuclei in the rat. Brain Res 562:230-242.

LeDoux JE, Moscarello J, Sears R, Campese V (2017) The birth, death and resurrection of avoidance: a reconceptualization of a troubled paradigm. Mol Psychiatry 22:24-36.

López DE, Saldaña E, Nodal FR, Merchán MA, Warr WB (1999) Projections of cochlear root neurons, sentinels of the rat auditory pathway. J Comp Neurol 415:160-174.

Mena-Segovia J, Bolam JP (2017) Rethinking the pedunculopontine nucleus: from cellular organization to function. Neuron 94:7-18.
Mena-Segovia J, Bolam JP, Magill PJ (2004) Pedunculopontine nucleus and basal ganglia: distant relatives or part of the same family? Trends Neurosci 27:585-588.

Mineka S (1979) The role of fear in theories of avoidance learning, flooding, and extinction. Psychol Bull 86:985-1010.

Mori F, Okada KI, Nomura T, Kobayashi Y (2016) The pedunculopontine tegmental nucleus as a motor and cognitive interface between the cerebellum and basal ganglia. Front Neuroanat 10:109.

Mowrer OH (1960) Learning theory and behavior. New York: Wiley.

Nodal FR, Lopez DE (2003) Direct input from cochlear root neurons to pontine reticulospinal neurons in albino rat. J Comp Neurol 460:80-93.

Pan WX, Hyland BI (2005) Pedunculopontine tegmental nucleus controls conditioned responses of midbrain dopamine neurons in behaving rats. J Neurosci 25:4725-4732.

Peek MY, Card GM (2016) Comparative approaches to escape. Curr Opin Neurobiol 41:167-173.

Pisanello M, Pisano F, Hyun M, Maglie E, Balena A, De Vittorio M, Sabatini BL, Pisanello F (2019) The three-dimensional signal collection field for fiber photometry in brain tissue. Front Neurosci 13:82.

Pisano F, Pisanello M, Lee SJ, Lee J, Maglie E, Balena A, Sileo L, Spagnolo B, Bianco M, Hyun M, De Vittorio M, Sabatini BL, Pisanello F (2019) Depth-resolved fiber photometry with a single tapered optical fiber implant. Nat Methods 16:1185-1192.

Ramirez F, Moscarello JM, LeDoux JE, Sears RM (2015) Active avoidance requires a serial basal amygdala to nucleus accumbens shell circuit. J Neurosci 35:3470-3477.

Reese NB, Garcia-Rill E, Skinner RD (1995) Auditory input to the pedunculopontine nucleus: II. Unit responses. Brain Res Bull 37:265-273.

Roseberry TK, Lee AM, Lalive AL, Wilbrecht L, Bonci A, Kreitzer AC (2016) Cell-type-specific control of brainstem locomotor circuits by basal ganglia. Cell 164:526-537.

Ryczko D, Dubuc R (2013) The multifunctional mesencephalic locomotor region. Curr Pharm Des 19:4448-4470.

Schofield BR, Motts SD (2009) Projections from auditory cortex to cholinergic cells in the midbrain tegmentum of guinea pigs. Brain Res Bull 80:163-170

Terburg D, Scheggia D, Triana Del Rio R, Klumpers F, Ciobanu AC, Morgan B, Montoya ER, Bos PA, Giobellina G, van den Burg EH, de Gelder B, Stein DJ, Stoop R, van Honk J (2018) The basolateral amygdala is essential for rapid escape: a human and rodent study. Cell 175:723-735. e716.

Tovote P, Esposito MS, Botta P, Chaudun F, Fadok JP, Markovic M, Wolff SB, Ramakrishnan C, Fenno L, Deisseroth K, Herry C, Arber S, Luthi A (2016) Midbrain circuits for defensive behaviour. Nature 534:206-212.

Westby GW, Keay KA, Redgrave P, Dean P, Bannister M (1990) Output pathways from the rat superior colliculus mediating approach and avoidance have different sensory properties. Exp Brain Res 81:626-638.

Xiong XR, Liang F, Zingg B, Ji XY, Ibrahim LA, Tao HW, Zhang LI (2015) Auditory cortex controls sound-driven innate defense behaviour through corticofugal projections to inferior colliculus. Nat Commun 6:7224.

Yilmaz M, Meister M (2013) Rapid innate defensive responses of mice to looming visual stimuli. Curr Biol 23:2011-2015.

Yoo JH, Zell V, Wu J, Punta C, Ramajayam N, Shen X, Faget L, Lilascharoen V, Lim BK, Hnasko TS (2017) Activation of pedunculopontine glutamate neurons is reinforcing. J Neurosci 37:38-46. 